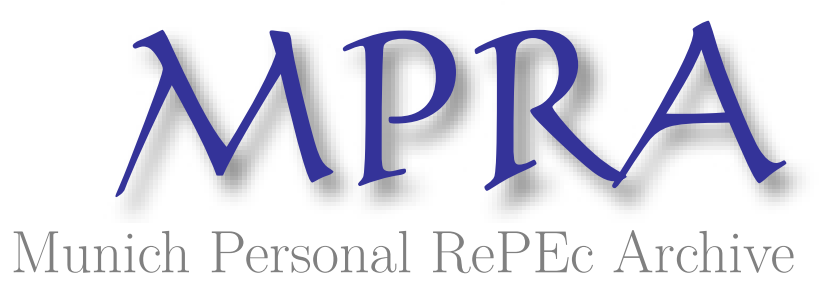

\title{
A New Exploration of Optimal IPR Protection and International Policy Cooperation
}

Dai, Darong and Shen, Kunrong

Department of Economics, School of Business, Nanjing University

18 September 2011

Online at https://mpra.ub.uni-muenchen.de/40039/

MPRA Paper No. 40039, posted 13 Jul 2012 14:15 UTC 


\title{
A New Exploration of Optimal IPR Protection and International Policy Cooperation
}

\author{
Darong Dai ${ }^{\mathrm{a}, 1} \quad$ Kunrong Shen $^{\mathrm{b}}$ \\ ${ }^{\mathrm{a}, \mathrm{b}}$ Department of Economics, School of Business, Nanjing University, Nanjing 210093,
} P. R. China

\begin{abstract}
In this paper, a variety-expanding growth model has been constructed in the classical North-South framework. Optimal degree of IPR protection has been explicitly computed both for the North and the South in the sense of welfare maximizing, in the short run and non-short run of the economies. Endogenous innovation growth rate and the policy of innovation subsidy for the Southern government have been introduced into our model. The relationship between IPR protection and long-term growth rate has also been thoroughly investigated. Finally, the issue about international policy cooperation has been explored and we show that it is possible that an optimal degree of Southern IPR protection would also be globally efficient in some comparatively weak conditions.
\end{abstract}

JEL classification: F42; H24; O31; O34; O38.

Keywords: Optimal IPR protection; Subsidy to innovation; Endogenous innovation growth; Technology match; North; South; International policy cooperation.

\footnotetext{
${ }^{1}$ Corresponding author. E-mail: daidarong998@163.com. Helpful comments from Jian Li are gratefully acknowledged. Any remaining errors are, of course, our own responsibility. We are grateful to National Natural Science Fund of China (71073076) and Major Bid Project of National Social Science Fund of China (07\&ZD009) for financial support. It is also a periodically academic result of a subproject titled by Economic Growth and Structural Transformation Research which is funded by Transformation and Development Research of Nanjing University, an innovation basis of philosophy and social science of National Education Department.
} 


\section{INTRODUCTION}

Our major goal of this paper is to investigate the existence of an optimal degree of intellectual property rights (IPR) protection for the developing country in the classical North-South framework. In our general equilibrium model, the South invents new intermediates as well as the North, and the imitation, which only exists in the South, will be divided into two types ${ }^{2}$ : the efficient imitation, that is imitating the intermediates invented in the North to improve the final output of the South, and the inefficient imitation, i.e., imitating other domestic innovators. It is easy to notice that a strengthening of IPR protection will exhibit both positive and negative effects on the South, given an equal (e.g., without discrimination) treatment to the above two kinds of imitations. Then, naturally, the problem facing us is that whether there is an optimal degree of IPR protection for the South after comprehensively balancing between these fundamentally opposing objects.

Rather, another policy instrument, subsidy to innovation, for the South has been introduced into our model, which will actually relax the tension ${ }^{3}$ facing the Southern government. And the optimal degree of IPR protection in the sense of welfare maximizing has also been computed and studied, both in the short run and non-short run of the economies. Moreover, we have comparatively and thoroughly explored the case of exogenous innovation and that of endogenous innovation ${ }^{4}$. Finally, the issue of international policy cooperation has been explored, and we, as a matter of fact, prove that there are possibilities such that the corresponding optimal degree of Southern IPR protection is also globally efficient in some conditions.

Generally speaking, some literatures insist that any IPR protection in the South

\footnotetext{
2 In Chu et al (2011)'s Schumpeterian growth model of distance to frontier, imitation activities also consist of two types, efficient imitation and inefficient imitation, and they call it efficient imitation because it raises the level of technology in the industry and call it inefficient imitation because it contributes nothing to the industry's level of technology. Noting that our model is the classical expanding-variety growth model, our definition of the two kinds of imitation activities is a little different from Chu et al (2011)'s, and one can refer to section 2.10. of our following model for more details.

${ }^{3}$ The purpose of the Southern government is, on the one hand, encouraging domestic innovation while, on the other hand, promoting economic growth with domestic welfare improved, which indeed benefits from efficient imitation. Noting that, the Southern government, after subsidy policy being incorporated into our model, can employ effective policy combination, which would thus enhance the policy flexibility, and hence the tension is, to some extent, relaxed. And one can refer to section 2.15. of the following model for details.

${ }^{4}$ Specifically, we realize endogenous innovation by means of "endogenous innovation growth rate", which would be regarded as a reasonable approach.
} 
will hurt the South; while, alternatively, other papers argued that there is an optimal level of IPR protection for the South. To the former, for example, Chin and Grossman (1990) conducted their partial equilibrium analysis in a North-South trading environment with exclusion of any possibility of innovation in the South, finding that the North always benefits from having the protection of intellectual property rights while the South benefits from the ability to pirate technology. Deardorff (1992) studied the welfare effects of global patent protection and suggested that the welfare of the inventing country certainly rises with the extension of patent protection while that of the other country probably falls. Schneider (2005)'s analysis suggests that IPRs have a stronger impact on domestic innovation for developed counties and might even negatively impact innovation in developing countries. And also, in his seminal paper, Helpman (1993) develops a dynamic general equilibrium framework in which the North invents new products and the South imitates them, proving that the South will lose from tighter intellectual property rights in the short run of the economies. Nevertheless, to the best of our knowledge, most of the subsequent literatures inspired by Helpman (1993) prove that there should exist an optimal degree of IPR protection in both quality-ladder models and expanding-variety models.

For instance, after noting that $R \& D$ is easier in competitive sectors than it is in monopolistic sectors for both technical and legal reasons, Horri and Iwaisako (2007) argued that there is an optimal degree of IPR protection so that the long-term growth rate is maximized. In an expanding-variety type R\&D-based endogenous growth model, Kwan and Lai (2003) show that there is a tradeoff between current loss in consumption and future gain in growth rate when IPR protection is strengthened across industries, thereby proving that there exists a finite optimal degree of IPR protection.

What's more, Furukawa (2007) constructs a variety expansion model of endogenous growth in a closed economy, and shows that there is an inverted U-shaped relationship between innovation and IPR protection, thereby implying that IPR protection is not always the stronger the better. Chen and Puttitanun (2005) illustrate the trade-off between imitating foreign technologies and encouraging 
domestic innovation in a developing country's choice of IPRs and their empirical analysis confirms that there is a U-shaped relationship between IPRs and economic development. And inspired by the Chinese experience, Chu et al. (2011) develop a Schumpeterian growth model of distance to frontier and show that optimal IPR protection is stage-dependent, that is, at an early stage of development, the country implements weak IPR protection to facilitate imitation, while at a later stage of development, the country implements strong IPR protection to encourage domestic innovation ${ }^{5}$.

In the present paper, it is shown that there is an inverted U-shaped relationship between Northern welfare and the inverse measure of Southern IPR protection, which resembles to Helpman (1993)'s conclusion that tighter IPR protection also hurts the North whenever economies begin in steady state with certain small rates of imitation. Moreover, after incorporating endogenous innovation growth rate into our model, we prove that there is a U-shaped relationship between Northern equilibrium growth rate and Southern IPR protection. On the other hand, for the South, we show that, given the specification of lump-sum taxation, an increasing of the subsidy rate to innovation will definitely improve the welfare. We argue that there always exists an optimal degree of Southern IPR protection in our model and there are complementary relationships between those optimal IPR protection policies and the innovation subsidy policy. Finally, it is confirmed that there is a U-shaped relationship between equilibrium growth rate and the IPR protection, and the equilibrium growth rate of the economy does not depend on innovation subsidy, after innovation growth rate being endogenously concerned.

Furthermore, there are also some papers that study the issue of international policy cooperation, for example, Grossman and Lai (2004) studied the policy game between the North and South, proving that the harmonization of patent policies is neither necessary nor sufficient for the efficiency of the global patent regime when two governments have an incentive to negotiate an international patent agreement.

\footnotetext{
${ }^{5}$ Our discussion corresponds to a later stage of development. However, our results reveal that IPR protection is not always the stronger the better both for the South and the North.
} 
However, our concerning of international policy cooperation, via noting that only Southern IPR protection is discussed, is a little different from that of Grossman and Lai (2004), and actually, we argue the existence of an optimal degree of Southern IPR protection, which is explicitly computed, and which is globally efficient.

What's the main inspiration of the present paper? Firstly, a simple framework is supplied so that the optimal degree of IPR protection can be explicitly computed, which will completely supports very rich comparative static analyses. Secondly, some papers (Spencer and Brander, 1983; Glass, 1997; Muniagurria and Singh, 1997; Haaland and Kind, 2008) have studied the efficient employment of subsidy policy, nevertheless, few, if any, like the present paper thoroughly depicts the internal relationship between optimal IPR protection and the policy choice of innovation subsidy. We, accordingly, claim that a good understanding of the internal interaction mechanisms between different policies would greatly improve the efficiency of corresponding policy combinations in reality. It is especially worth emphasizing is that the case of endogenous innovation growth would lead to totally different conclusions relative to that of exogenous innovation growth. And hence we insist, as is shown in our model, that the above two cases should be comparatively and thoroughly studied, thereby offering insights into our recognition about institutional arrangements in reality. Moreover, the role of technology match has been discussed in determining the optimal IPR protection for the South in the North-South framework. And finally, the present paper is encouraged to supply a simple approach leading to a new ${ }^{6}$ understanding of international policy cooperation.

The rest of the paper is organized as follows: section 2 presents the model and our key propositions, section 3 discusses the issue of international policy cooperation conditional on the model in section 2, section 4 concludes and the Appendix provides

\footnotetext{
${ }^{6}$ It is new because it is based upon the specification that there is only one control variable, which belongs to the South and intrinsically affects the North, and which is also endogenously impacted or partly determined by parameters belong to the North. And thus the way of discussing international policy cooperation is different from that of existing literatures, e.g., Kehoe (1989), Maggi (1999), Devereux and Engel (2003), and Canzoneri et al (2005). And especially, Kehoe (1989) referred to the international cooperative regime in the sense of that policies are set sequentially by a single decision-making body to maximize world welfare. Certainly, in the current paper we don't discuss such kind of cooperative game which intimately depends on the existence of a so-called world welfare function. Moreover, our discussion expands following two steps: firstly, the existence of optimal IPR protection both for the South and the North; and secondly, the possibilities of international policy cooperation.
} 
the main mathematical derivations.

\section{THE MODEL}

In the classical North-South framework, the model is naturally divided into two parts, the North and the South, which are, nevertheless, linked by such activities that the South will imitate Northern innovation while with the Southern government faced with the problem to choose certain degree of IPR protection with purpose, on the one hand, of promoting economic growth with welfare improved, and, on the other hand, encouraging domestic innovation and respecting Trade-Related Aspects of Intellectual Property Rights (TRIPs). Moreover, in each part of the model, endogenous innovation growth rate is concerned with its resulting consequences being thoroughly discussed.

\section{The North}

\subsection{Final Production}

The final good sector employs labor and a set of intermediate goods as inputs. The technology for producing final good is represented by the following constant returns to scale production function,

$$
Y^{N}(t)=\beta^{N}\left(L^{N}\right)^{1-\alpha^{N}} \int_{0}^{A^{N}(t)} x^{N}(j)^{\alpha^{N}} d j,
$$

where $\beta^{N}>0$, an exogenously given constant, represents productivity parameter, $x^{N}(j)$ is the quantity of the intermediate $\operatorname{good} j$ with $j \in\left[0, A^{N}(t)\right], A^{N}(t)$ is the number of intermediate goods available at period $t, L^{N}$ is labor supply and is assumed to be fixed over time, and $\alpha^{N} \in(0,1)$ denotes the share of intermediate machines. As is shown in Furukawa (2007), this specification describes different inputs as imperfect substitutes, implying that no intermediate good is intrinsically better or worse than any other, irrespective of the time of introduction.

\subsection{Imitation Process}

As in the model of Helpman (1993), in order to investigate the consequences of a 
tightening of IPR protection in the South, the costless imitation process reads as follows,

$$
\dot{A}^{c_{1}, S}(t)=\mu^{S}\left[A^{N}(t)-A^{c_{1}, S}(t)\right]
$$

in which $0 \leq \mu^{S}<\infty$ denotes the imitation rate or the hazard rate at which each product that has not been imitated yet is going to be imitated at the next date and hence a tightening of IPR protection in the South implies a decline of $\mu^{S}$, i.e., $\mu^{S}$ is an inverse measure of IPR protection. Obviously, $\mu^{S}$ may be impacted and even determined by some other factors. However, we just, for simplicity and in order to effectively capture the economic intuition behind our model, focus those factors on certain degree of IPR protection. $A^{c_{1}, S}(t)\left(\leq A^{N}(t)\right)$ denotes the number of intermediates that have already been imitated by the South up to period $t$. And it is assumed that once an intermediate is imitated, it will become competitive.

\subsection{Intermediate Sector}

The intermediate good sector consists of monopolistically competitive firms, and technology is symmetric across varieties, that is, the production of one unit of intermediate good requires one unit of final good, which is assumed to be the numeraire. However, after the costless imitation process being introduced into the model, and hence once a product is imitated, the monopoly power of the original producer is lost and its price is pushed down to the marginal cost by competition. Accordingly, the prices of intermediate goods amount to,

$$
p^{N}(j)= \begin{cases}1, & j \in\left[0, A^{c_{1}, S}(t)\right] \\ 1 / \alpha^{N}, & j \in\left(A^{c_{1}, S}(t), A^{N}(t)\right]\end{cases}
$$

And the corresponding demand functions are given by,

$$
x^{N}(j)= \begin{cases}x^{c_{1}, N} \equiv\left(\beta^{N}\right)^{1 /\left(1-\alpha^{N}\right)} L^{N}\left(\alpha^{N}\right)^{1 /\left(1-\alpha^{N}\right)}, & j \in\left[0, A^{c_{1}, S}(t)\right] \\ x^{m, N} \equiv\left(\beta^{N}\right)^{1 /\left(1-\alpha^{N}\right)} L^{N}\left(\alpha^{N}\right)^{2 /\left(1-\alpha^{N}\right)}, & j \in\left(A^{c_{1}, S}(t), A^{N}(t)\right]\end{cases}
$$

And it is easy to see that the aggregate demand for intermediates can be expressed as follows, 


$$
X^{N}(t) \equiv \int_{0}^{A^{N}(t)} x^{N}(j) d j=x^{c_{1}, N}\left\{A^{c_{1}, S}(t)+\left(\alpha^{N}\right)^{1 /\left(1-\alpha^{N}\right)}\left[A^{N}(t)-A^{c_{1}, S}(t)\right]\right\},
$$

\subsection{Innovation Sector}

We introduce, following Helpman (1993), the following innovation process,

$$
A^{N}(t)=A^{N}(0) e^{g^{N} t}
$$

which implies that the North introduces new intermediates at a constant exogenous rate $g^{N}$. And thus it is easily seen that the fraction of goods that have not been imitated by the South reads as,

$$
\zeta^{N}(t)=\left[A^{N}(t)-A^{c_{1}, S}(t)\right] / A^{N}(t),
$$

which evolves in accordance with the following differential equation,

$$
\dot{\zeta}^{N}(t)=g^{N}-\left(g^{N}+\mu^{S}\right) \zeta^{N}(t),
$$

And the solution of the ordinary differential equation (ODE) amounts to,

$$
\zeta^{N}(t)=\bar{\zeta}^{N}+\left[\zeta^{N}(0)-\bar{\zeta}^{N}\right] e^{-\left(g^{N}+\mu^{S}\right) t}, \quad \bar{\zeta}^{N}=g^{N} /\left(g^{N}+\mu^{S}\right)
$$

where $\bar{\zeta}^{N}$ denotes the long-run steady state and $\zeta^{N}(0)$ represents the exogenously given initial condition. Provided that developing a new variety requires $b^{N}$ units of final good, and noting that in the next time interval of length $d t$, innovators that have not previously been imitated face the probability $\mu^{S} d t$ ( $d t$ is sufficiently small such that $\left.\mu^{S} d t \in[0,1]\right)$ of being imitated, and thus the standard no arbitrage condition of the asset markets can be expressed as follows,

$$
r^{N}(t) V^{N}(t)-\dot{V}^{N}(t)=\pi^{N}(t)-\mu^{S} V^{N}(t),
$$

which would be regarded as a Bellman type equation, where $r^{N}$ is the nominal interest rate, $V^{N}$ is the value of the innovator, and $\pi^{N}$ is the monopolistic profits in the differentiated intermediates market. Furthermore, the free entry condition in the R\&D markets implies that the present discounted value (PDV) of profits from innovation cannot exceed the entry $\operatorname{cost} b^{N}$, i.e., $V^{N}(t)=b^{N}$. Hence, the nominal interest rate is 
constant over time and could be expressed as follows,

$$
\begin{aligned}
& r^{N}=r^{m, N}-\mu^{S}, \\
& r^{m, N} \equiv \pi^{N} / b^{N}=\frac{\left(1-\alpha^{N}\right)\left(\alpha^{N}\right)^{\left(1+\alpha^{N}\right) /\left(1-\alpha^{N}\right)}\left(\beta^{N}\right)^{1 /\left(1-\alpha^{N}\right)} L^{N}}{b^{N}},
\end{aligned}
$$

where $r^{m, N}$ denotes the instantaneous rate of return for innovation.

\subsection{Market Clearing for Final Good}

As usual, the final good is used for consumption, for production of intermediate products, and also for innovation. Consequently, the market clearing condition for the final good can be written as,

$$
Y^{N}(t)=C^{N}(t)+X^{N}(t)+b^{N} \dot{A}^{N}(t),
$$

where $b^{N}$ denotes the entry cost of $\mathrm{R} \& \mathrm{D}$ markets, $C^{N}$ represents the aggregate consumption and $X^{N}(t)$ is given in (5). Inserting the demand functions in (4) into (1) produces,

$$
Y^{N}(t)=\left(\beta^{N}\right)^{1 /\left(1-\alpha^{N}\right)} L^{N}\left(\alpha^{N}\right)^{\alpha^{N} /\left(1-\alpha^{N}\right)}\left\{A^{c_{1}, S}(t)+\left(\alpha^{N}\right)^{\alpha^{N} /\left(1-\alpha^{N}\right)}\left[A^{N}(t)-A^{c_{1}, S}(t)\right]\right\},
$$

Substituting (5), (6) and (11) into (10), then the following expression of aggregate consumption is immediately derived,

$$
\begin{aligned}
C^{N}(t)= & \left(\beta^{N}\right)^{1 /\left(1-\alpha^{N}\right)} L^{N}\left\{\left[\left(\alpha^{N}\right)^{\alpha^{N} /\left(1-\alpha^{N}\right)}-\left(\alpha^{N}\right)^{1 /\left(1-\alpha^{N}\right)}\right]\left(1-\zeta^{N}(t)\right) A^{N}(t)\right. \\
& \left.+\left[\left(\alpha^{N}\right)^{2 \alpha^{N} /\left(1-\alpha^{N}\right)}-\left(\alpha^{N}\right)^{2 /\left(1-\alpha^{N}\right)}\right] \zeta^{N}(t) A^{N}(t)\right\}-b^{N} g^{N} A^{N}(t),
\end{aligned}
$$

Hence, we obtain,

$$
C^{N}(0)=\eta_{1}^{N} \zeta^{N}(0)+\eta_{2}^{N},
$$

Where

$$
\begin{aligned}
\eta_{1}^{N} \equiv & \left(\beta^{N}\right)^{1 /\left(1-\alpha^{N}\right)} L^{N} A^{N}(0)\left\{\left[\left(\alpha^{N}\right)^{2 \alpha^{N} /\left(1-\alpha^{N}\right)}-\left(\alpha^{N}\right)^{2 /\left(1-\alpha^{N}\right)}\right]\right. \\
& \left.-\left[\left(\alpha^{N}\right)^{\alpha^{N} /\left(1-\alpha^{N}\right)}-\left(\alpha^{N}\right)^{1 /\left(1-\alpha^{N}\right)}\right]\right\}<0, \\
\eta_{2}^{N} \equiv & \left(\beta^{N}\right)^{1 /\left(1-\alpha^{N}\right)} L^{N} A^{N}(0)\left[\left(\alpha^{N}\right)^{\alpha^{N} /\left(1-\alpha^{N}\right)}-\left(\alpha^{N}\right)^{1 /\left(1-\alpha^{N}\right)}\right]-b^{N} g^{N} A^{N}(0),
\end{aligned}
$$

Noting that $C^{N}(0) \geq 0$, hence $\eta_{2}^{N}>0$. 


\subsection{Representative Agent}

Without loss of generality, we assume that the abstract general equilibrium economy is populated by infinitely lived agent who derives utility from consumption and supplies inelastic labor. Agent is supposed to exhibit log preferences ${ }^{7}$ with perfect foresight and the corresponding dynamic maximization problem is given by,

$$
\begin{aligned}
& \max _{\left\{C^{N}(t)_{t \geq 0}\right.} U^{N}=\int_{0}^{\infty} e^{-\rho^{N} t} \ln C^{N}(t) d t, \\
& \text { s.t. } \quad \dot{B}^{N}(t)=r^{N} B^{N}(t)+w^{N}(t) L^{N}-C^{N}(t),
\end{aligned}
$$

in which $\rho^{N}>0$ is the subjective discount factor, $r^{N}$ is given in (9), $B^{N}$ is the value of asset holdings, $L^{N}$ is the constant labor supply and $w^{N}$ is the equilibrium wage rate. Thus, the optimality condition, i.e., the standard Euler equation of consumption reads as follows,

$$
\gamma_{C}^{N} \equiv \frac{\dot{C}^{N}(t)}{C^{N}(t)}=r^{m, N}-\mu^{S}-\rho^{N},
$$

in which $r^{m, N}$ is defined in (9') and $\gamma_{C}^{N}$ also denotes the equilibrium growth rate of the model economy. And as expected, the growth rate in (16) is a decreasing function of imitation rate thanks to the limited duration of the monopoly, thereby effectively reducing the private value of each innovation. Therefore, we conclude, only from the viewpoint of long-run economic growth, that a tightening of IPR protection in the South will benefit the North.

\subsection{The Welfare Effects of Southern IPR Protection to the North}

Notice from the previous section that,

$$
U^{N}=\int_{0}^{\infty} e^{-\rho^{N} t} \ln C^{N}(t) d t=\frac{\ln C^{N}(0)}{\rho^{N}}+\frac{\gamma_{C}^{N}}{\left(\rho^{N}\right)^{2}},
$$

where $C^{N}(0)$ is given by (13). So if $\zeta^{N}(0) \neq \bar{\zeta}^{N}$, i.e., the economy does not start from

\footnotetext{
${ }^{7}$ It is easy to see that the specification of preference structure will not fundamentally change our following conclusions and hence choosing log preferences here is just for simplicity and convenience.
} 
the steady state, thus we get,

$$
\frac{\partial U^{N}}{\partial \mu^{S}}=-\frac{1}{\left(\rho^{N}\right)^{2}}<0
$$

Then we claim,

PROPOSITION 1. Given above specifications, and if $\zeta^{N}(0) \neq \bar{\zeta}^{N}$, then a tightening of intellectual property rights in the South, that is, a decline of the imitation rate, will exhibit positive welfare effect to the North.

On the other hand, we restrict the analysis to economies that are initially in steady state; namely, $\zeta^{N}(0)=\bar{\zeta}^{N}=g^{N} /\left(g^{N}+\mu^{S}\right)$, then in view of (13) and (14) shows that,

$$
\frac{\partial C^{N}(0)}{\partial \mu^{S}}=-\frac{\eta_{1}^{N} g^{N}}{\left(g^{N}+\mu^{S}\right)^{2}}>0,
$$

And so, by (17), the following first order condition immediately,

$$
\begin{aligned}
& \frac{\partial U^{N}}{\partial \mu^{S}}=\frac{1}{\rho^{N} C^{N}(0)} \frac{\partial C^{N}(0)}{\partial \mu^{S}}-\frac{1}{\left(\rho^{N}\right)^{2}}=0, \\
& \Leftrightarrow \eta_{2}^{N}\left(g^{N}+\mu^{S}\right)^{2}+\eta_{1}^{N} g^{N}\left(g^{N}+\mu^{S}\right)+\eta_{1}^{N} g^{N} \rho^{N}=0,
\end{aligned}
$$

Thus, we obtain,

$$
\mu_{1}^{*}=\frac{-\eta_{1}^{N} g^{N}+\sqrt{\left(\eta_{1}^{N} g^{N}\right)^{2}-4 \eta_{2}^{N} \eta_{1}^{N} g^{N} \rho^{N}}}{2 \eta_{2}^{N}}-g^{N},
$$

Moreover, noting by (14) that,

$$
\frac{\partial^{2} U^{N}}{\partial\left(\mu^{S}\right)^{2}}=-\frac{1}{\rho^{N}\left[C^{N}(0)\right]^{2}}\left[\frac{\partial C^{N}(0)}{\partial \mu^{S}}\right]^{2}+\frac{1}{\rho^{N} C^{N}(0)} \frac{\partial^{2} C^{N}(0)}{\partial\left(\mu^{S}\right)^{2}}<0,
$$

Therefore, we conclude that,

PROPOSITION 2. Based on the above assumptions, then in the short run of the economy, there must exist an optimal level of IPR protection $\mu_{1}^{*}$, which is defined in (18) and is independent of $A^{N}(0)$, such that the welfare of the North is maximized.

REMARK. We have proved that there exists an optimal degree of IPR protection in the South such that the welfare of the North is maximized in certain conditions, which are rather weak. That is to say, there is an inverted U-shaped relationship between the 
welfare function of the North and the inverse measure of IPR protection in the South. In other words, there is a critical value of $\mu^{S}$ denoted by $\mu_{1}^{*}$ defined in (18), and when $\mu^{S}<\mu_{1}^{*}$, a tightening of IPR protection will hurt the North but when $\mu^{S}>\mu_{1}^{*}$, a tightening of IPR protection will improve the welfare of the North.

Finally, a comparison of Proposition 1 and Proposition 2 shows that, under certain conditions, the short-run welfare effect of IPR protection in the South would be totally different from the other non-short-run, say, midterm-run or long-run, welfare effects. In particular, a tightening of IPR protection may factually improve the long-run welfare of the North while reducing its short-run welfare.

\subsection{Endogenous Innovation Growth Rate}

First of all, we still employ the following innovation process,

$$
A^{N}(t)=A^{N}(0) e^{g^{N} t}
$$

Then, assume that developing $\dot{A}^{N}(t)$ new varieties requires $\widehat{b^{N}(t)}$ units of final good. And, in particular, $\widehat{b^{N}(t)}$ is given by,

$$
\widehat{b^{N}(t)} \equiv \frac{\left(g^{N}\right)^{\sigma^{N}+1}}{\left(\sigma^{N}+1\right) v^{N}} A^{N}(t),
$$

where $\sigma^{N}>1$ is an exogenously given constant such that $\partial^{2} \widehat{b^{N}(t)} / \partial\left(g^{N}\right)^{2}>0$ and it is in line with the classical specification of cost function in microeconomics, $\partial \widehat{b^{N}(t)} / \partial A^{N}(t)>0$ reflects the fact that existing innovations will to some extent make new innovation more difficult ${ }^{8}$, and $v^{N}>0$ denotes the productivity parameter, including accumulation of experience following from learning by doing (e.g., Arrow, 1962; Young, 1993; Stein, 1997) and "standing on the shoulder of giants" (see, Scotchmer, 1991) and so on. All in all, the specification reveals the idea or the fact that technological process or technical innovation will play a critical role in

\footnotetext{
${ }^{8}$ Segerstrom (1998) specifically uses similar specification to successfully remove out the so-called "scale effects" noted and analyzed by Jones (1995) in R\&D-based models.
} 
maintaining barriers to entry and therefore impacting market structure (see, Levin, 1978). And market structure in turn will greatly shape the technological processes. Thus, the expected profit maximization problem facing the innovator is defined as follows,

$$
\max _{0<g^{N}<1}\left(1-\mu^{S} d t\right) \dot{A}^{N}(t) \pi^{N}(t)-\widehat{b^{N}(t)}
$$

where $\left(1-\mu^{S} d t\right)$ denotes the probability of not being imitated in the next $d t$ length of time interval. Solving the static maximization problem yields,

$$
g^{N}=\left[\left(\beta^{N}\right)^{1 /\left(1-\alpha^{N}\right)}\left(1-\alpha^{N}\right)\left(\alpha^{N}\right)^{\left(1+\alpha^{N}\right) /\left(1-\alpha^{N}\right)} L^{N} v^{N}\left(1-\mu^{S} d t\right)\right]^{1 / \sigma^{N}},
$$

from which we see that $\partial g^{N} / \partial \mu^{S}<0$, and just in this sense of (19), we claim that innovation growth rate $g^{N}$ is endogenously determined. And since developing a new variety requires $\widehat{b^{N}(t)} / \dot{A}^{N}(t)$ units of final good. Thus, combining (9) with (9'), the following nominal interest rate is derived,

$$
\begin{aligned}
& r^{N}=r^{m, N}-\mu^{S} \\
& r^{m, N} \equiv \pi^{N} \dot{A}^{N}(t) / \widehat{b^{N}(t)}=\frac{\left(1-\alpha^{N}\right)\left(\alpha^{N}\right)^{\left(1+\alpha^{N}\right) /\left(1-\alpha^{N}\right)}\left(\sigma^{N}+1\right) v^{N}\left(\beta^{N}\right)^{1 /\left(1-\alpha^{N}\right)} L^{N}}{\left(g^{N}\right)^{\sigma^{N}}}
\end{aligned}
$$

where $r^{m, N}$ denotes the instantaneous rate of return for innovation. Notice from (16) that the standard Euler equation of the representative consumer is expressed as,

$$
\gamma_{C}^{N} \equiv \frac{\dot{C}^{N}(t)}{C^{N}(t)}=r^{m, N}-\mu^{S}-\rho^{N},
$$

To make the notations more compact, we put,

$$
\begin{aligned}
& r^{m, N}=\eta_{3}^{N} /\left(g^{N}\right)^{\sigma^{N}}, \\
& \eta_{3}^{N} \equiv\left(1-\alpha^{N}\right)\left(\alpha^{N}\right)^{\left(1+\alpha^{N}\right) /\left(1-\alpha^{N}\right)}\left(\sigma^{N}+1\right) v^{N}\left(\beta^{N}\right)^{1 /\left(1-\alpha^{N}\right)} L^{N},
\end{aligned}
$$

And,

$$
\begin{aligned}
& g^{N}=\left[\eta_{4}^{N}\left(1-\mu^{S} d t\right)\right]^{1 / \sigma^{N}}, \\
& \eta_{4}^{N} \equiv\left(1-\alpha^{N}\right)\left(\alpha^{N}\right)^{\left(1+\alpha^{N}\right) /\left(1-\alpha^{N}\right)} v^{N}\left(\beta^{N}\right)^{1 /\left(1-\alpha^{N}\right)} L^{N},
\end{aligned}
$$

Notice that $\eta_{3}^{N}=\left(\sigma^{N}+1\right) \eta_{4}^{N}$. And thus we can easily get, 


$$
\frac{\partial \gamma_{C}^{N}}{\partial \mu^{S}}=\left(\sigma^{N}+1\right)\left(\eta_{4}^{N}\right)^{2}\left(g^{N}\right)^{-2 \sigma^{N}} d t-1=0 \Leftrightarrow \mu^{S}=\frac{1-\sqrt{\left(\sigma^{N}+1\right) d t}}{d t},
$$

Where

$$
\frac{1-\sqrt{\left(\sigma^{N}+1\right) d t}}{d t}>0 \Leftrightarrow d t<\frac{1}{\sigma^{N}+1},
$$

Therefore, we conclude the following proposition,

PROPOSITION 3. Given the endogenous innovation growth rate defined in (19), there is a critical value of the inverse measure of IPR protection, $\mu^{S}=\left[1-\sqrt{\left(\sigma^{N}+1\right) d t}\right] / d t$, provided $d t<1 /\left(\sigma^{N}+1\right)$ so that,

$$
\frac{\partial \gamma_{C}^{N}}{\partial \mu^{S}} \lesseqgtr 0 \Leftrightarrow\left\{\begin{array}{l}
\mu^{S} \lesseqgtr\left[1-\sqrt{\left(\sigma^{N}+1\right) d t}\right] / d t \\
d t<1 /\left(\sigma^{N}+1\right)
\end{array}\right.
$$

That is, there is a U-shaped relationship between the Northern equilibrium growth rate and the Southern IPR protection.

REMARK. It follows from (16) that a strengthening of Southern IPR protection will always promote the long-run economic growth of the North. However, after innovation growth rate being endogenously concerned, there is a U-shaped relationship between the Northern equilibrium growth rate and the Southern IPR protection, i.e., a tightening of Southern IPR protection may inevitably retard the Northern economic growth, due to Proposition 3.

Moreover, noting that if $\zeta^{N}(0)=\bar{\zeta}^{N}$, then by (19),

$$
\frac{\partial \zeta^{N}(0)}{\partial \mu^{S}}=\frac{\mu^{S}}{\left(g^{N}+\mu^{S}\right)^{2}} \frac{\partial g^{N}}{\partial \mu^{S}}-\frac{g^{N}}{\left(g^{N}+\mu^{S}\right)^{2}}<0,
$$

Hence, by (13), (14), (15) and (19), and noting that $b^{N}=\widehat{b^{N}(t)} / \dot{A}^{N}(t)$,

$$
\frac{\partial C^{N}(0)}{\partial \mu^{S}}=\eta_{1}^{N} \frac{\partial \zeta^{N}(0)}{\partial \mu^{S}}-\frac{A^{N}(0)}{v^{N}}\left(g^{N}\right)^{\sigma^{N}} \frac{\partial g^{N}}{\partial \mu^{S}}>0
$$

On the other hand, if $\zeta^{N}(0) \neq \bar{\zeta}^{N}$, then,

$$
\frac{\partial C^{N}(0)}{\partial \mu^{S}}=-\frac{A^{N}(0)}{v^{N}}\left(g^{N}\right)^{\sigma^{N}} \frac{\partial g^{N}}{\partial \mu^{S}}>0
$$


So from (17) we see that,

$$
\frac{\partial U^{N}}{\partial \mu^{S}}=\frac{1}{\rho^{N} C^{N}(0)} \frac{\partial C^{N}(0)}{\partial \mu^{S}}+\frac{1}{\left(\rho^{N}\right)^{2}} \frac{\partial \gamma_{C}^{N}}{\partial \mu^{S}}>0,
$$

holds true for both $\zeta^{N}(0)=\bar{\zeta}^{N}$ and $\zeta^{N}(0) \neq \bar{\zeta}^{N}$ provided $\partial \gamma_{C}^{N} / \partial \mu^{S}>0$

Therefore, combining with Proposition 3, we get,

PROPOSITION 4. If $\mu^{S} \geq\left[1-\sqrt{\left(\sigma^{N}+1\right) d t}\right] / d t$ with $d t<1 /\left(\sigma^{N}+1\right)$, then we easily get $\partial U^{N} / \partial \mu^{S}>0$ for both $\zeta^{N}(0)=\bar{\zeta}^{N}$ and $\zeta^{N}(0) \neq \bar{\zeta}^{N}$, that is, a tightening of IPR protection in the South, i.e., a decreasing of $\mu^{S}$, will reduce the welfare of the North.

REMARK. Interesting should it be to notice that Helpman (1993) shows that tighter intellectual property rights also hurt the North whenever economies begin in steady state with small rates of imitation, however, it is shown, after the innovation growth rate being endogenously concerned, that a tightening of Southern IPR protection will definitely hurt the North when the imitation rate is above one critical value whenever the economies begin in steady state or begin in non-steady state. Moreover, it would be remarkable that, after incorporating endogenous innovation growth rate into the current model, a comparison of Proposition 4 with Proposition 1 and Proposition 2 shows that the Northern welfare effects with respect to Southern choice of IPR protection would be totally different. And we may further claim that endogenous innovation growth rate in the North would result in a much subtler relationship between Southern IPR protection and Northern welfare.

\section{The South}

\subsection{Final Good Production}

Different from the North, here we specify the following production function for the competitive firms,

$$
Y^{S}(t)=\beta^{S}\left(L^{S}\right)^{1-\alpha^{S}}\left[\int_{0}^{A^{S}(t)} x^{S}(j)^{\alpha^{S}} d j+\int_{0}^{A^{c, S}(t)} \Theta^{1-\alpha^{S}} z^{S}(j)^{\alpha^{S}} d j\right],
$$

where $\beta^{S}>0$ is the productivity parameter, $x^{S}(j)$ is the quantity of the intermediate 
$\operatorname{good} j, j \in\left[0, A^{S}(t)\right]$, while $z^{S}(j)$ is the quantity of the intermediate good $j$, $j \in\left[0, A^{c_{1}, S}(t)\right], \quad A^{c_{1}, S}(t)$ is defined in (2) and $A^{S}(t)$ is the number of intermediate goods invented by Southern domestic firms up to time $t, L^{N}$ is constant labor supply and $\alpha^{S} \in(0,1)$ denotes the share of intermediate products. What's more, $\Theta \in[0,1]$ is an exogenous parameter that reflects the match level of intermediate $j, j \in\left[0, A^{c_{1}, S}(t)\right]$, to the final production. And hence, rather, we take $x^{S}(j)$ and $z^{S}(j)$ as the corresponding quantities of different (e.g., in the sense of production technology) intermediates which belong to $\left[0, A^{S}(t)\right]$ and $\left[0, A^{c_{1}, S}(t)\right]$, respectively. Moreover, the exponent on $\Theta$ is just for computation convenience.

\subsection{Imitation Process}

Besides the costless imitation process introduced in (2), we define the following another costless imitation process,

$$
\dot{A}^{c_{2}, S}(t)=\mu^{S}\left[A^{S}(t)-A^{c_{2}, S}(t)\right]
$$

where $0 \leq \mu^{S}<\infty$ represents the hazard rate and also is an inverse measure of IPR protection. $A^{c_{2}, S}(t)$ is the number of intermediates invented by firms in the South that have already been imitated by other domestic firms. And as is specified in the North, once an intermediate good is imitated, it will become competitive.

REMARK. It is mentioned in Introduction that the Southern imitation has been naturally divided into two types, efficient imitation and inefficient imitation. It is especially worth emphasizing here is that the standard of efficiency is not in the sense of consumer welfare but just in the sense of final output. Therefore, it is natural, via combining (22) and (23), to see that the imitation activities introduced in (23) are actually inefficient, whereas the imitation activities shown in (2) are efficient and their efficiency level depends on the parameter $\Theta \in[0,1]$. 


\subsection{Intermediate Sector}

With concerning of the imitation process introduced in previous section, the intermediate products can be naturally divided into two groups: the imitated, $\left[0, A^{c_{2}, S}(t)\right]$, and the monopolized, $\left(A^{c_{2}, S}(t), A^{S}(t)\right]$. Therefore, based on the technology assumption that one unit of intermediate good requires one unit of final good, assumed to be the numeraire, the optimal quantities and prices set, according to the principle that marginal revenue equals marginal cost, by the monopolists are,

$$
\begin{aligned}
& x^{S}(j)= \begin{cases}x^{c_{2}, S} \equiv\left(\beta^{S}\right)^{1 /\left(1-\alpha^{S}\right)} L^{S}\left(\alpha^{S}\right)^{1 /\left(1-\alpha^{S}\right)}, & j \in\left[0, A^{c_{2}, S}(t)\right] \\
x^{m, S} \equiv\left(\beta^{S}\right)^{1 /\left(1-\alpha^{S}\right)} L^{S}\left(\alpha^{S}\right)^{2 /\left(1-\alpha^{S}\right)}, & j \in\left(A^{c_{2}, S}(t), A^{S}(t)\right]\end{cases} \\
& z^{S}(j)=z^{c_{1}, S} \equiv \Theta\left(\beta^{S}\right)^{1 /\left(1-\alpha^{S}\right)} L^{S}\left(\alpha^{S}\right)^{1 /\left(1-\alpha^{S}\right)}, \quad j \in\left[0, A^{c_{1}, S}(t)\right]
\end{aligned}
$$

and,

$$
p^{S}(j)= \begin{cases}1, & j \in\left[0, A^{c_{2}, S}(t)\right] \\ 1 / \alpha^{S}, & j \in\left(A^{c_{2}, S}(t), A^{S}(t)\right] \\ 1, & j \in\left[0, A^{c_{1}, S}(t)\right]\end{cases}
$$

respectively. Although we take $j \in\left[0, A^{c_{1}, S}(t)\right]$ and $j \in\left[0, A^{c_{2}, S}(t)\right]$ as totally different intermediates from the viewpoint of technology match to the final production and hence output contribution, we treat them equally in prices as is shown in (26) since they are all competitive in the same markets ${ }^{9}$, from which the prices in (26) would be regarded as, to some extent, reasonable and acceptable. And most importantly, this assumption is far from being playing a determinant role in the current model. So the aggregate demand for intermediates amounts to,

$$
\begin{aligned}
X^{S}(t) & \equiv \int_{0}^{A^{S}(t)} x^{S}(j) d j+\int_{0}^{A^{c_{1}, S}(t)} z^{S}(j) d j \\
& =\left(\beta^{S}\right)^{1 /\left(1-\alpha^{S}\right)} L^{S}\left(\alpha^{S}\right)^{1 /\left(1-\alpha^{S}\right)}\left\{A^{c_{2}, S}(t)+\left(\alpha^{S}\right)^{1 /\left(1-\alpha^{S}\right)}\left[A^{S}(t)-A^{c_{2}, S}(t)\right]+\Theta A^{c_{1}, S}(t)\right\},
\end{aligned}
$$

\subsection{Innovation Sector}

\footnotetext{
${ }^{9}$ In particular, they share the same marginal cost, i.e., one unit of final good. And thus, there are not any arbitrage opportunities in the Southern imitation sector. Moreover, even though they don't share the same marginal cost initially, long-run competition (perfect competition assumption) definitely pulls back the economy to stay in an equilibrium without any arbitrage opportunities, therefore, we, initially, without loss of generality, suppose that the economy is just lying in the above long-run equilibrium.
} 
As for the North, we employ the following innovation process ${ }^{10}$,

$$
A^{S}(t)=A^{S}(0) e^{g^{S} t}
$$

where $A^{S}(0)>0$ denotes the initial level of innovation and $g^{S}$ is an exogenously given growth rate. The fraction of intermediate goods that have not been imitated can thus be characterized, following Helpman (1993), by the following differential equation,

$$
\dot{\zeta}^{S}(t)=g^{S}-\left(g^{S}+\mu^{S}\right) \zeta^{S}(t)
$$

And its solution is given as,

$$
\zeta^{S}(t)=\bar{\zeta}^{S}+\left[\zeta^{S}(0)-\bar{\zeta}^{S}\right] e^{-\left(g^{S}+\mu^{S}\right) t}, \bar{\zeta}^{S}=g^{S} /\left(g^{S}+\mu^{S}\right)
$$

where $\bar{\zeta}^{s}$ denotes the long-run steady state of the dynamic system. Davidson and Segerstrom (1998) present an endogenous growth model and find that only innovative R\&D subsidies lead to faster economic growth while imitative R\&D subsidies actually lead to slower economic growth. Hence, we, in line with Davidson and Segerstrom (1998), only incorporate subsidy policy into innovation sector and thus suppose that developing a new variety requires $\left(1-\tau_{A^{S}}\right) b^{S}{ }^{11}$, in which $\tau_{A^{s}} \in(0,1)$ denotes the subsidy rate to innovation from the Southern government, units of final good. And the standard no arbitrage condition for the asset markets can be written as,

$$
\frac{\pi^{S}(t)}{V^{S}(t)}+\frac{\dot{V}^{S}(t)}{V^{S}(t)}=r^{S}(t)+\mu^{S}+\phi^{S}, 0<\phi^{S}<1,
$$

where $r^{S}$ is the nominal interest rate, $\phi^{S}$ denotes the risk cost induced by the immaturity of the Southern financial markets, $V^{S}$ is the value of the innovator, and $\pi^{S}$ is the monopolistic profits in the differentiated intermediates market. Then, using the free entry condition in the R\&D markets, the nominal interest rate reads as,

$$
r^{S}=r^{m, S}-\mu^{S}-\phi^{S},
$$

\footnotetext{
${ }^{10}$ Some papers (see Howitt (1999) for instance) assume that there are two kinds of innovation, horizontal and vertical, in their models. Here we only consider horizontal innovation due to our specification of the model. ${ }^{11}$ As is shown in existing literatures (e.g., Spence, 1984; Spencer and Brander, 1983), we also employ cost-reducing innovation subsidy.
} 


$$
r^{m, S}=\frac{\left(1-\alpha^{S}\right)\left(\alpha^{S}\right)^{\left(1+\alpha^{S}\right) /\left(1-\alpha^{S}\right)}\left(\beta^{S}\right)^{1 /\left(1-\alpha^{S}\right)} L^{S}}{\left(1-\tau_{A^{S}}\right) b^{S}}
$$

where $r^{m, S} \equiv \pi^{S} /\left(1-\tau_{A^{S}}\right) b^{S}$ denotes the instantaneous rate of return for innovation and it is an increasing function of the subsidy rate to innovation.

\subsection{Market Clearing for the Final Good}

Notice that the final good is used for consumption, for production of intermediate machines, and for innovation, the market clearing condition can therefore be written as,

$$
Y^{S}(t)=C^{S}(t)+X^{S}(t)+\left(1-\tau_{A^{S}}\right) b^{S} \dot{A}^{S}(t)
$$

in which $C^{S}$ represents the aggregate consumption and $X^{S}(t)$ is given in (27). Substituting (24) and (25) into (22) produces,

$$
Y^{S}(t)=\left(\beta^{S}\right)^{1 /\left(1-\alpha^{S}\right)} L^{S}\left(\alpha^{S}\right)^{\alpha^{S} /\left(1-\alpha^{S}\right)}\left\{A^{c_{2}, S}(t)+\left(\alpha^{S}\right)^{\alpha^{S} /\left(1-\alpha^{S}\right)}\left[A^{S}(t)-A^{c_{2}, S}(t)\right]+\Theta A^{c_{1}, S}(t)\right\}
$$

And so substituting (27), (28) and (35) into (34) yields,

$$
\begin{aligned}
C^{S}(t)= & \left(\beta^{S}\right)^{1 /\left(1-\alpha^{S}\right)} L^{S}\left\{\left[\left(\alpha^{S}\right)^{2 \alpha^{S} /\left(1-\alpha^{S}\right)}-\left(\alpha^{S}\right)^{2 /\left(1-\alpha^{S}\right)}\right]-\left[\left(\alpha^{S}\right)^{\alpha^{S} /\left(1-\alpha^{S}\right)}-\left(\alpha^{S}\right)^{1 /\left(1-\alpha^{S}\right)}\right]\right\} \\
& \times \zeta^{S}(t) A^{S}(t)-\Theta\left(\beta^{S}\right)^{1 /\left(1-\alpha^{S}\right)} L^{S}\left[\left(\alpha^{S}\right)^{\alpha^{S} /\left(1-\alpha^{S}\right)}-\left(\alpha^{S}\right)^{1 /\left(1-\alpha^{S}\right)}\right] \zeta^{N}(t) A^{N}(t) \\
& +\left(\beta^{S}\right)^{1 /\left(1-\alpha^{S}\right)} L^{S}\left[\left(\alpha^{S}\right)^{\alpha^{S} /\left(1-\alpha^{S}\right)}-\left(\alpha^{S}\right)^{1 /\left(1-\alpha^{S}\right)}\right]\left[A^{S}(t)+\Theta A^{N}(t)\right] \\
& -\left(1-\tau_{A^{S}}\right) b^{S} g^{S} A^{S}(t)
\end{aligned}
$$

Then, we obtain,

$$
C^{S}(0)=\eta_{1}^{S} \zeta^{S}(0)+\eta_{2}^{S} \zeta^{N}(0)+\eta_{3}^{S}-\eta_{4}^{S}\left(1-\tau_{A^{S}}\right),
$$

Where

$$
\begin{aligned}
\eta_{1}^{S} \equiv & \left(\beta^{S}\right)^{1 /\left(1-\alpha^{S}\right)} L^{S} A^{S}(0)\left\{\left[\left(\alpha^{S}\right)^{2 \alpha^{S} /\left(1-\alpha^{S}\right)}-\left(\alpha^{S}\right)^{2 /\left(1-\alpha^{S}\right)}\right]\right. \\
& \left.-\left[\left(\alpha^{S}\right)^{\alpha^{S} /\left(1-\alpha^{S}\right)}-\left(\alpha^{S}\right)^{1 /\left(1-\alpha^{S}\right)}\right]\right\}<0, \\
\eta_{2}^{S} \equiv & \Theta\left(\beta^{S}\right)^{1 /\left(1-\alpha^{S}\right)} L^{S} A^{N}(0)\left[\left(\alpha^{S}\right)^{1 /\left(1-\alpha^{S}\right)}-\left(\alpha^{S}\right)^{\alpha^{S} /\left(1-\alpha^{S}\right)}\right]<0,
\end{aligned}
$$




$$
\begin{aligned}
& \eta_{3}^{S} \equiv\left(\beta^{S}\right)^{1 /\left(1-\alpha^{S}\right)} L^{S}\left[\left(\alpha^{S}\right)^{\alpha^{S} /\left(1-\alpha^{S}\right)}-\left(\alpha^{S}\right)^{1 /\left(1-\alpha^{S}\right)}\right]\left[A^{S}(0)+\Theta A^{N}(0)\right]>0, \\
& \eta_{4}^{S} \equiv b^{S} g^{S} A^{S}(0)>0
\end{aligned}
$$

Hence, we get,

$$
C^{S}(0)=\underbrace{\eta_{1}^{S} \zeta^{S}(0)}_{\leq 0}+\underbrace{\eta_{2}^{S} \zeta^{N}(0)}_{\leq 0}+\underbrace{\eta_{3}^{S}}_{>0}+\underbrace{-\eta_{4}^{S}\left(1-\tau_{A^{S}}\right)}_{<0},
$$

\subsection{Representative Agent}

As usual, the dynamic maximization problem for the representative agent is given as,

$$
\begin{aligned}
& \max _{\left\{C^{S}(t)\right\}_{t \geq 0}} U^{S}=\int_{0}^{\infty} e^{-\rho^{S} t} \ln C^{S}(t) d t, \\
& \text { s.t. } \quad \dot{B}^{S}(t)=r^{S} B^{S}(t)+w^{S}(t) L^{S}-C^{S}(t)-T^{S},
\end{aligned}
$$

where $\rho^{S}>0$ is the subjective discount factor, $B^{S}$ is the value of asset holdings, $w^{S}$ is the equilibrium wage rate, and $T^{S}$ is the lump-sum tax. Thus, the standard Euler equation of consumption reads as follows,

$$
\gamma_{C}^{S} \equiv \frac{\dot{C}^{S}(t)}{C^{S}(t)}=r^{m, S}-\mu^{S}-\phi^{S}-\rho^{S},
$$

from which we find that, just from the point of view of long-run economic growth, both a tightening of IPR protection and an increasing of the rate of innovation subsidy, provided the lump-sun taxation and ceteris paribus, will benefit the South ${ }^{12}$.

REMARK. Noting that our theme is not optimal taxation theory, we only introduce lump-sum tax into our model as the unique resource of governmental subsidy to innovation. However, it is predictable that the discussion of optimal innovation subsidy will immediately become very complicated after incorporating both government balanced budget constraint and some other linear taxes, say, income tax and consumption tax, into the present model.

\footnotetext{
${ }^{12}$ Gould and Gruben (1996), indeed, find that stronger intellectual property rights protection corresponds to higher economic growth rates in a cross-country sample.
} 


\subsection{Government}

In this section, specifically, both $g^{N}$ and $g^{S}$ are assumed to be exogenously given. It follows from (37) and (43) that, the welfare (or utility) function of the South can be expressed as,

$$
U^{S}=\int_{0}^{\infty} e^{-\rho^{S} t} \ln C^{S}(t) d t=\frac{\ln C^{S}(0)}{\rho^{S}}+\frac{\gamma_{C}^{S}}{\left(\rho^{S}\right)^{2}},
$$

Hence, if $\zeta^{S}(0) \neq \bar{\zeta}^{S}$ and $\zeta^{N}(0) \neq \bar{\zeta}^{N}$, then,

$$
\frac{\partial U^{S}}{\partial \mu^{S}}=-\frac{1}{\left(\rho^{S}\right)^{2}}<0
$$

If $\zeta^{S}(0) \neq \bar{\zeta}^{S}$ and $\zeta^{N}(0)=\bar{\zeta}^{N}$, then by (7), (37), (39) and (44), we have the following first order condition (FOC),

$$
\begin{aligned}
\frac{\partial U^{S}}{\partial \mu^{S}} & =\frac{-\eta_{2}^{S} g^{N}}{\rho^{S} C^{S}(0)\left(g^{N}+\mu^{S}\right)^{2}}-\frac{1}{\left(\rho^{S}\right)^{2}}=0, \\
& \Leftrightarrow \eta_{5}^{S}\left(g^{N}+\mu^{S}\right)^{2}+\eta_{2}^{S} g^{N}\left(g^{N}+\mu^{S}\right)+\eta_{2}^{S} g^{N} \rho^{S}=0 .
\end{aligned}
$$

where $\eta_{5}^{S} \equiv \eta_{1}^{S} \zeta^{S}(0)+\eta_{3}^{S}-\eta_{4}^{S}\left(1-\tau_{A^{S}}\right)$. Noting that $C^{S}(0) \geq 0$ and thus it is easy to see that $\eta_{5}^{S}>0$ by (37'). Thus, we have,

$$
\mu_{2}^{*}=\frac{-\eta_{2}^{S} g^{N}+\sqrt{\left(\eta_{2}^{S} g^{N}\right)^{2}-4 \eta_{2}^{S} g^{N} \eta_{5}^{S} \rho^{S}}}{2 \eta_{5}^{S}}-g^{N},
$$

whose optimality is fully ensured by the following second order condition,

$$
\frac{\partial^{2} U^{S}}{\partial\left(\mu^{S}\right)^{2}}=-\frac{1}{\rho^{S}\left[C^{S}(0)\right]^{2}}\left[\frac{\partial C^{S}(0)}{\partial \mu^{S}}\right]^{2}+\frac{2 \eta_{2}^{S} g^{N}}{\rho^{S} C^{S}(0)\left(g^{N}+\mu^{S}\right)^{3}}<0,
$$

by (39).If $\zeta^{S}(0)=\bar{\zeta}^{S}$ and $\zeta^{N}(0) \neq \bar{\zeta}^{N}$, then by (30), (37), (38) and (44), we get the following FOC,

$$
\begin{aligned}
\frac{\partial U^{S}}{\partial \mu^{S}} & =\frac{-\eta_{1}^{S} g^{S}}{\rho^{S} C^{S}(0)\left(g^{S}+\mu^{S}\right)^{2}}-\frac{1}{\left(\rho^{S}\right)^{2}}=0, \\
& \Leftrightarrow \eta_{6}^{S}\left(g^{S}+\mu^{S}\right)^{2}+\eta_{1}^{S} g^{S}\left(g^{S}+\mu^{S}\right)+\eta_{1}^{S} g^{S} \rho^{S}=0 .
\end{aligned}
$$

where $\eta_{6}^{S} \equiv \eta_{2}^{S} \zeta^{N}(0)+\eta_{3}^{S}-\eta_{4}^{S}\left(1-\tau_{A^{S}}\right)>0$. Noting that $C^{S}(0) \geq 0$ and thus $\eta_{6}^{S}>0$ by 
(37'). Therefore, we obtain,

$$
\mu_{3}^{*}=\frac{-\eta_{1}^{S} g^{S}+\sqrt{\left(\eta_{1}^{S} g^{S}\right)^{2}-4 \eta_{1}^{S} g^{S} \eta_{6}^{S} \rho^{S}}}{2 \eta_{6}^{S}}-g^{S},
$$

And meanwhile noting from (38) that,

$$
\frac{\partial^{2} U^{S}}{\partial\left(\mu^{S}\right)^{2}}=-\frac{1}{\rho^{S}\left[C^{S}(0)\right]^{2}}\left[\frac{\partial C^{S}(0)}{\partial \mu^{S}}\right]^{2}+\frac{2 \eta_{1}^{S} g^{S}}{\rho^{S} C^{S}(0)\left(g^{S}+\mu^{S}\right)^{3}}<0,
$$

Moreover, if $\zeta^{S}(0)=\bar{\zeta}^{S}$ and $\zeta^{N}(0)=\bar{\zeta}^{N}$, then by (7), (30), (37), (38), (39) and (44), we get the following FOC,

$$
\frac{-\eta_{1}^{S} g^{S}}{C^{S}(0)\left(g^{S}+\mu^{S}\right)^{2}}+\frac{-\eta_{2}^{S} g^{N}}{C^{S}(0)\left(g^{N}+\mu^{S}\right)^{2}}=\frac{1}{\rho^{S}},
$$

If $g^{N}=g^{S}$, then we get,

$$
\eta_{7}^{S}\left(g^{S}+\mu^{S}\right)^{2}+\left(\eta_{1}^{S}+\eta_{2}^{S}\right) g^{S}\left(g^{S}+\mu^{S}\right)+\left(\eta_{1}^{S}+\eta_{2}^{S}\right) g^{S} \rho^{S}=0,
$$

where $\eta_{7}^{S} \equiv \eta_{3}^{S}-\eta_{4}^{S}\left(1-\tau_{A^{s}}\right)$, and it follows from (37') that $\eta_{7}^{S}>0$ always holds true. Hence, we obtain,

$$
\mu_{4}^{*}=\frac{-\left(\eta_{1}^{S}+\eta_{2}^{S}\right) g^{S}+\sqrt{\left(\eta_{1}^{S}+\eta_{2}^{S}\right)^{2}\left(g^{S}\right)^{2}-4\left(\eta_{1}^{S}+\eta_{2}^{S}\right) g^{S} \eta_{7}^{S} \rho^{S}}}{2 \eta_{7}^{S}}-g^{S},
$$

Further notice that, by (38), (39), and $g^{N}=g^{S}$, then the following second order condition is clearly fulfilled,

$$
\frac{\partial^{2} U^{S}}{\partial\left(\mu^{S}\right)^{2}}=\frac{-1}{\rho^{S}\left[C^{S}(0)\right]^{2}}\left[\frac{\partial C^{S}(0)}{\partial \mu^{S}}\right]^{2}+\frac{2\left(\eta_{1}^{S}+\eta_{2}^{S}\right) g^{S}}{\rho^{S} C^{S}(0)\left(g^{S}+\mu^{S}\right)^{3}}<0,
$$

Accordingly, we conclude that,

PROPOSITION 5. (i) If $\zeta^{S}(0) \neq \bar{\zeta}^{S}$ and $\zeta^{N}(0) \neq \bar{\zeta}^{N}$, then a tightening of intellectual property rights protection will improve the welfare of the South.

(ii) $\operatorname{If} \zeta^{S}(0) \neq \bar{\zeta}^{S}, \zeta^{N}(0)=\bar{\zeta}^{N}$, then there is an optimal level of IPR protection given in (45) for the South.

(iii) If $\zeta^{S}(0)=\bar{\zeta}^{S}, \zeta^{N}(0) \neq \bar{\zeta}^{N}$, then there exists an optimal level of IPR protection given in (46) for the South. 
(iv) $\operatorname{If} \zeta^{S}(0)=\bar{\zeta}^{S}, \zeta^{N}(0)=\bar{\zeta}^{N}$ and $g^{N}=g^{S}$, thus we see that there is an optimal degree of IPR protection, defined in (47), for the South.

REMARK. Proposition 5(i) shows that a strengthening of IPR protection will definitely improve the welfare of the South, given exogenous innovation growth, in the non-short run of the economies. It is, one the one hand, different from the point insisted by Chin and Grossman (1990), Deardorff (1992) and Schneider (2005) that a tightening of IPR protection will surely hurt the South, and on the other hand, different from the illustration of the existence of an optimal degree of IPR protection shown in Kwan and Lai (2003), Furukawa (2007) and Horri and Iwaisako (2007). Thus, we argue, regardless of the specificity of model specification, that we have provided the conditions under which a tightening of IPR protection will definitely improve the Southern welfare in general expanding-variety models and in the classical North-South framework.

Helpman (1993), provided that the South just imitates the new products invented by the North, proved that the South will lose from tighter intellectual property rights in the short run of the economies, however, after introducing Southern innovation activities into the framework resembles to Helpman (1993), Proposition 5(iv) shows conditions based upon which there is an optimal degree of IPR protection, i.e., the South may gain from tighter intellectual property rights, in the short run of the economies.

On the other hand, notice that, by (33), (37), (41) and (44),

$$
\frac{\partial U^{S}}{\partial \tau_{A^{S}}}=\frac{\eta_{4}^{S}}{\rho^{S} C^{S}(0)}+\frac{r^{m, S}}{\left(\rho^{S}\right)^{2}\left(1-\tau_{A^{S}}\right)}>0,
$$

Therefore, we summarize,

PROPOSITION 6. Given the specification of lump-sum taxation, an increasing of the subsidy rate to innovation will definitely improve the welfare of the South.

REMARK. The economic intuition of this Proposition can be summarized as follows: first, lump-sum taxation exhibits no distortion effects and hence consumption process is not affected for given initial level of consumption; second, an increasing of 
the subsidy rate to innovation implies an increasing of intermediates provided there is not any "crowding-out effect", and the quantity of efficient imitation would keep unchanged, if not increased, for given level of technology match $\Theta$ and IPR protection in the short-run or midterm-run of the economy, therefore, final output, i.e., national income, would definitely increase. And hence budget constraint line may probably move out (ceteris paribus) for a given increasing of lump-sum tax, which, rather, supports an increasing of consumption and so welfare via combining with the first point mentioned above.

Moreover, we obtain the following proposition,

PROPOSITION 7. Given the optimal degree of IPR protection $\mu_{2}^{*}$ defined in (45), we have $\partial \mu_{2}^{*} / \partial \tau_{A^{s}}<0$ and $\partial \mu_{2}^{*} / \partial g^{S}>0$, that is to say, there is complementarity between the optimal degree of IPR protection and the subsidy rate to innovation, and the smaller of $g^{S}$ the tighter of optimal degree of IPR protection in the South, respectively.

PROOF. See Appendix A.

REMARK. For the Southern government, the dual $\left(\mu_{2}^{*}, \tau_{A^{s}}\right)$ would be an efficient policy combination thanks to Proposition 7. And we insist that Proposition 7 indeed throws light on our understanding of policy or institutional choice of the South. Firstly, noting that a decreasing of $g^{S}$ implies a decreasing of domestic innovation for any given initial level $A^{S}(0)$, the corresponding optimal degree of IPR protection hence should be higher via noting that one goal of the South is encouraging domestic innovation. However, a tightening of IPR protection will reduce its efficient imitation activities by noting the fact that $\mu^{S}$ does not affect $A^{N}(t)$ when $g^{N}$ and $A^{N}(0)$ are exogenously given constants. Thus, a tightening of IPR protection, by combining the above two opposing effects, may and also may not reduce the final output or national income of the South. However, Proposition 7 clearly confirms that the former positive effect will dominate the later negative effect (ceteris paribus) of a given tightening of IPR protection, i.e., a decreasing of $g^{s}$ inevitably implies a strengthening of IPR 
protection.

For $\mu_{3}^{*}$, the following proposition is derived,

PROPOSITION 8. Given the existence of the optimal degree of IPR protection $\mu_{3}^{*}$ defined in (46), then we get $\partial \mu_{3}^{*} / \partial \tau_{A^{s}}<0$ and $\partial \mu_{3}^{*} / \partial \Theta<0$, that is, there is complementarity between the optimal degree of IPR protection and the subsidy rate to innovation, and the higher the level of technology match the tighter the optimal IPR protection, respectively.

PROOF. See Appendix B.

Moreover, we obtain the following proposition,

PROPOSITION 9. Conditional on the existence of the optimal degree of IPR protection $\mu_{4}^{*}$ defined in (47), then we get $\partial \mu_{4}^{*} / \partial \tau_{A^{s}}<0$, that is, there is complementarity between the optimal degree of IPR protection and the subsidy rate to innovation.

PROOF. See Appendix C.

REMARK. For policy-makers, capturing the internal reactive mechanisms between different policy variables should be of great importance. Proposition 7, Proposition 8 and Proposition 9 consistently show that the policy instruments for Southern government are not independent of each other, and particularly, the optimal degree of IPR protection is endogenously determined by the rate of innovation subsidy $\tau_{A^{s}}$. That is to say, $\tau_{A^{s}}$ not only directly acts on the equilibrium growth rate of the economy but also indirectly acts on the equilibrium growth rate via directly acting on $\mu^{S}$. Now, suppose that $\mu_{2}^{*}\left(\right.$ or $\left.\mu_{3}^{*}, \mu_{4}^{*}\right)$ seems somewhat large due to other parameters ${ }^{13}$ such as $\Theta, g^{S}$ and $g^{N}$, etc, hence the Southern government can effectively decrease the value of $\mu_{2}^{*}\left(\right.$ or $\left.\mu_{3}^{*}, \mu_{4}^{*}\right)$ (ceteris paribus) by a sufficient increasing of $\tau_{A^{s}}$, and vise verse. And just in this case and in this sense, we insist, as is argued in Introduction, that subsidy policy would effectively relax the tension facing the Southern government.

\footnotetext{
${ }^{13}$ These parameters are not control variables for the government.
} 


\subsection{Endogenous Innovation Growth Rate}

First, we still use the following innovation process,

$$
A^{S}(t)=A^{S}(0) e^{g^{S} t}
$$

Then, suppose that developing $\dot{A}^{S}(t)$ new varieties requires $\left(1-\tau_{A^{s}}\right) \widehat{b^{S}(t)}$ units of final goods. And, in particular, $\widehat{b^{S}(t)}$ is defined as,

$$
\widehat{b^{S}(t)} \equiv \frac{\left(g^{S}\right)^{\sigma^{S}+1}}{\left(\sigma^{S}+1\right) v^{S}} A^{S}(t)
$$

where $\sigma^{S}>1$ is an exogenously given constant, and $v^{S}$ denotes the productivity parameter. Therefore, the static maximization problem facing the innovator is given by,

$$
\max _{0<g^{S}<1}\left(1-\mu^{S} d t\right) \dot{A}^{S}(t) \pi^{S}(t)-\left(1-\tau_{A^{S}}\right) \widehat{b^{S}(t)},
$$

where $\left(1-\mu^{S} d t\right)$ represents the probability of not being imitated by other domestic firms in the next $d t$ length of time interval. Solving the static maximization problem gives,

$$
g^{S}=\left[\frac{\left(\beta^{S}\right)^{1 /\left(1-\alpha^{S}\right)}\left(1-\alpha^{S}\right)\left(\alpha^{S}\right)^{\left(1+\alpha^{S}\right) /\left(1-\alpha^{S}\right)} L^{S} v^{S}\left(1-\mu^{S} d t\right)}{1-\tau_{A^{S}}}\right]^{1 / \sigma^{S}},
$$

from which we see that $g^{S}$ is an increasing function ${ }^{14}$ of $\tau_{A^{s}}$ and meanwhile a decreasing function of $\mu^{S}$, and just in this sense of (48), we call $g^{S}$ the endogenous innovation growth rate. As is required, developing a new variety employs $\left(1-\tau_{A^{S}}\right) \widehat{b^{S}(t)} / \dot{A}^{S}(t)$ units of final good. Thus, the nominal interest rate can be rewritten as,

$$
r^{S}=r^{m, S}-\mu^{S}
$$

\footnotetext{
14 The point worth emphasizing here is that government subsidization of R\&D should invoke "additionality effect" while it is also possible that it will "crowd out" private financing of R\&D (e.g., Görg and Strobl, 2007). Therefore, the innovation growth rate may and may not be an increasing function of governmental subsidy rate. To make things easier, here in the present paper we don't take the so-called "crowding-out effect" into account.
} 


$$
r^{m, S}=\frac{\left(1-\alpha^{S}\right)\left(\alpha^{S}\right)^{\left(1+\alpha^{S}\right) /\left(1-\alpha^{S}\right)}\left(\sigma^{S}+1\right) v^{S}\left(\beta^{S}\right)^{1 /\left(1-\alpha^{S}\right)} L^{S}}{\left(1-\tau_{A^{S}}\right)\left(g^{S}\right)^{\sigma^{S}}}
$$

where $r^{m, S}$ denotes the instantaneous rate of return for innovation. And note from (43) that the standard Euler equation for the representative agent reads as,

$$
\gamma_{C}^{S} \equiv \frac{\dot{C}^{S}(t)}{C^{S}(t)}=r^{m, S}-\mu^{S}-\phi^{S}-\rho^{S},
$$

To make the notations more compact, we put,

$$
\begin{aligned}
& r^{m, S}=\eta_{9}^{S} /\left(1-\tau_{A^{S}}\right)\left(g^{S}\right)^{\sigma^{S}}, \\
& \eta_{9}^{S} \equiv\left(1-\alpha^{S}\right)\left(\alpha^{S}\right)^{\left(1+\alpha^{S}\right) /\left(1-\alpha^{S}\right)}\left(\sigma^{S}+1\right) v^{S}\left(\beta^{S}\right)^{1 /\left(1-\alpha^{S}\right)} L^{S},
\end{aligned}
$$

And,

$$
\begin{aligned}
& g^{S}=\left[\frac{\eta_{10}^{S}\left(1-\mu^{S} d t\right)}{1-\tau_{A^{S}}}\right]^{1 / \sigma^{S}}, \\
& \eta_{10}^{S} \equiv\left(1-\alpha^{S}\right)\left(\alpha^{S}\right)^{\left(1+\alpha^{S}\right) /\left(1-\alpha^{S}\right)} v^{S}\left(\beta^{S}\right)^{1 /\left(1-\alpha^{S}\right)} L^{S},
\end{aligned}
$$

So we find that $\eta_{9}^{S}=\left(\sigma^{S}+1\right) \eta_{10}^{S}$. Notice that,

$$
\frac{\partial g^{S}}{\partial \mu^{S}}=\frac{-\eta_{10}^{S} d t}{\sigma^{S}\left(1-\tau_{A^{S}}\right)}\left(g^{S}\right)^{1-\sigma^{S}}<0,
$$

Then,

$$
\frac{\partial \gamma_{C}^{S}}{\partial \mu^{S}}=\frac{\left(\sigma^{S}+1\right)\left(\eta_{10}^{S}\right)^{2} d t}{\left(1-\tau_{A^{S}}\right)^{2}\left(g^{S}\right)^{2 \sigma^{S}}}-1=0,
$$

which implies that,

$$
\mu^{S}=\frac{1-\sqrt{\left(\sigma^{S}+1\right) d t}}{d t},
$$

And since,

$$
\frac{\partial^{2} \gamma_{C}^{S}}{\partial\left(\mu^{S}\right)^{2}}=\frac{-2 \sigma^{S}\left(\sigma^{S}+1\right)\left(\eta_{10}^{S}\right)^{2} d t}{\left(1-\tau_{A^{S}}\right)^{2}\left(g^{S}\right)^{2 \sigma^{S}+1}} \frac{\partial g^{S}}{\partial \mu^{S}}>0,
$$

Accordingly, we summarize the following proposition,

PROPOSITION 10. Conditional on the endogenous innovation growth rate defined in (48), then there is a critical value of the inverse measure of IPR protection, 


$$
\begin{gathered}
\mu^{S}=\left[1-\sqrt{\left(\sigma^{S}+1\right) d t}\right] / d t, \text { provided that } d t<1 /\left(\sigma^{S}+1\right) \text { so that }, \\
\frac{\partial \gamma_{C}^{S}}{\partial \mu^{S}} \lesseqgtr 0 \Leftrightarrow\left\{\begin{array}{l}
\mu^{S} \lesseqgtr\left[1-\sqrt{\left(\sigma^{S}+1\right) d t}\right] / d t, \\
d t<1 /\left(\sigma^{S}+1\right),
\end{array}\right.
\end{gathered}
$$

That is, there is a U-shaped relationship between equilibrium growth rate and the IPR protection.

REMARK. It is worth noting that there should be a U-shaped relationship between equilibrium growth rate and the IPR protection after innovation growth rate being endogenously concerned while it follows from (43) that a tightening of IPR protection always implies an increasing of equilibrium growth rate. Proposition 10, indeed, is consistent with Chen and Puttitanun (2005)'s conclusion following from their empirical analysis with a panel data for 64 developing countries.

On the other hand, since,

$$
\frac{\partial g^{S}}{\partial \tau_{A^{s}}}=\frac{\eta_{10}^{S}\left(1-\mu^{S} d t\right)}{\left(1-\tau_{A^{s}}\right)^{2} \sigma^{S}}\left(g^{S}\right)^{1-\sigma^{s}}>0,
$$

Thus, by (48),

$$
\frac{\partial \gamma_{C}^{S}}{\partial \tau_{A^{S}}}=\frac{\eta_{9}^{S}}{\left(1-\tau_{A^{S}}\right)^{2}\left(g^{S}\right)^{\sigma^{S}}}\left[1-\frac{\eta_{10}^{S}\left(1-\mu^{S} d t\right)}{\left(1-\tau_{A^{S}}\right)\left(g^{S}\right)^{\sigma^{S}}}\right]=0,
$$

So we have,

PROPOSITION 11. Based on the endogenous innovation growth rate given by (48), then we get $\partial \gamma_{C}^{S} / \partial \tau_{A^{s}}=0$, i.e., the equilibrium growth rate of the economy is independent of the subsidy rate to innovation.

REMARK. Interesting should it be that, conditional on the endogenous innovation growth rate shown in (48), we see by Proposition 11 that the equilibrium growth rate is independent of the subsidy rate to innovation, which is in accord with the conclusion from Jones (1995) and Segerstrom (1998). Nonetheless, it is obviously shown by (43) that an increasing of the rate of innovation subsidy, provided the lump-sum taxation and ceteris paribus, will always imply a resulting increasing of equilibrium growth rate, which resembles to the results from Howitt $(1999,2000)$. To 
sum up, it is endogenous innovation growth that leads us from Howitt $(1999,2000)$ to Jones (1995) and Segerstrom (1998) in the current general expanding-variety model.

Moreover, we have,

PROPOSITION 12. Conditional on the endogenous innovation growth rate $g^{s}$ defined by (48), and if $\mu^{s} \geq\left[1-\sqrt{\left(\sigma^{S}+1\right) d t}\right] / d t$ with $d t<1 /\left(\sigma^{s}+1\right)$, then we always get $\partial U^{S} / \partial \mu^{S}>0$, i.e., a tightening of IPR protection would hurt the South.

PROOF. See Appendix D.

REMARK. A comparison of Proposition 5(i) and Proposition 12 shows that endogenous innovation growth will make the corresponding welfare effect of a tightening of IPR protection totally changed. And that's why we emphasize in the Introduction that the case of exogenous innovation growth and that of endogenous innovation growth should be comparatively studied.

Intuitively, by (44) we see that the welfare $U^{S}$ positively depends on the initial consumption $C^{S}(0)$ and the equilibrium growth rate of consumption $\gamma_{C}^{S}$. Provided that $\zeta^{S}(0) \neq \bar{\zeta}^{S}$ and $\zeta^{N}(0) \neq \bar{\zeta}^{N}$, thus, in the exogenous case, $C^{S}(0)$ is independent of $\mu^{S}$ by (37)-(41) with $\gamma_{C}^{S}$ negatively depends on $\mu^{S}$ by (43). So the marginal effect of $U^{S}$ with respect to $\mu^{S}$ will be negative, i.e., $\partial U^{S} / \partial \mu^{S}<0$. However, in the endogenous case, the proof of Proposition 12 shows that the initial consumption $C^{S}(0)$ always positively depends on $\mu^{S}$, i.e., a strengthening of IPR protection will definitively reduce the initial level of consumption. This may, unavoidably, sound strange and unbelievable ${ }^{15}$ even though it's not the focus here. The key point here is that endogenous innovation growth would probably result in $\partial \gamma_{C}^{S} / \partial \mu^{S}>0$. And it is this combining with $\partial C^{S}(0) / \partial \mu^{S}>0$ that inevitably leads us to the conclusion shown in Proposition 12. To further our analyses, the crucial point that induces the change from $\partial \gamma_{C}^{S} / \partial \mu^{S}<0$ to $\partial \gamma_{C}^{S} / \partial \mu^{S}>0$ totally bases on the

15 The relationship may be very ambiguous in reality. 
fact that $r^{m, S}$, instantaneous rate of return for innovation, now, positively depends on $\mu^{S}$ while in the exogenous case $r^{m, S}$ is just independent of $\mu^{S}$. As a matter of fact, we have the following logic diagram for endogenous innovation growth,

$$
\left.\mu^{S} \downarrow \Rightarrow g^{S} \uparrow \Rightarrow b^{S}=\widehat{b^{S}(t)} / \dot{A}^{N}(t) \uparrow \Rightarrow r^{m, S} \downarrow\left(\forall \text { given } \pi^{S}\right) \Rightarrow \gamma_{C}^{S} \downarrow \text { (ceteris paribus }\right)
$$

And that's the whole story. Obviously, the key point is “ $g^{S} \uparrow \Rightarrow b^{S}=\widehat{b^{S}(t)} / \dot{A}^{N}(t) \uparrow ”$, i.e., an increasing of the growth rate of innovation implies an increasing of average cost of innovation, which partly, if not completely, depends on our definition of $\widehat{b^{S}(t)}$. Noting that our definition of $\widehat{b^{S}(t)}$ is reasonable and acceptable in some sense. We argue that the current paper at least provides us with a "possibility", and given the logic behind the possibility, the above story inevitably happens.

\section{INTERNATIONAL POLICY COOPERATION}

In this section, we study the issue of policy cooperation between the North and the South. Note that an agreement of IPR protection cannot be achieved unless the incentive-problem has been solved firstly. Our purpose is to search for conditions under which a degree of IPR protection is optimal with respect to the welfare for both the North and the South. And as is shown that the welfare functions of the North and the South are maximized simultaneously, we call the corresponding degree of IPR protection in the South globally efficient.

As a matter of fact, we obtain,

Proposition 13. If $\rho^{S}=\rho^{N}, \Theta$ satisfies (E.1) ${ }^{16}$ and $A^{N}(0) / A^{S}(0)$ satisfies (E.4), then we get $\mu_{1}^{*}=\mu_{2}^{*}$. In particular, as $\zeta^{S}(0) \rightarrow 1$, and if $\rho^{S}=\rho^{N}, \Theta$ satisfies (E.1) and $A^{N}(0) / A^{S}(0)$ satisfies (E.5), then we get $\mu_{1}^{*}=\mu_{2}^{*}$.

PROOF. See Appendix E.

REMARK. It is $\mu_{1}^{*}$ and $\mu_{2}^{*}$ that are compared because they all depend on the same

${ }^{16}$ See Appendix E. 
condition $\zeta^{N}(0)=\bar{\zeta}^{N}$ by a view of Proposition 2 and Proposition 5(ii).

Moreover, we get,

PROPOSITION 14. If $\rho^{S}=\rho^{N}, A^{N}(0) / A^{S}(0)$ satisfies $(\mathrm{F} .3)^{17}$, then we get $\mu_{1}^{*}=\mu_{4}^{*}$.

PROOF. See Appendix F.

REMARK. It is $\mu_{1}^{*}$ and $\mu_{4}^{*}$ that are compared because they are conditional on the same assumption $\zeta^{N}(0)=\bar{\zeta}^{N}$ following from Proposition 2 and Proposition 5(iv). Moreover, we see, from the proofs of Proposition 13 and Proposition 14, that all the conditions are met as long as $\rho^{S}=\rho^{N}$ and $L^{S} \gg L^{N} \gg M>0$ for a given constant $M$. Actually, only $\rho^{S}=\rho^{N}$ is a binding requirement in reality, which, however, can be indeed relaxed through a little adjustment of the ratio $A^{N}(0) / A^{S}(0)$ in view of the definitions of $\mu_{1}^{*}, \mu_{2}^{*}$ and $\mu_{4}^{*}$. Therefore, we claim that: it follows from Proposition 13 jointly with Proposition 14 that international policy cooperation, given the welfare of the North and the South is maximized simultaneously ${ }^{18}$, is possible in some relatively weak conditions.

\section{CONCLUDING REMARKS}

In this paper, a variety expansion model has been constructed in the classical North-South framework. It is assumed that the South will invent new intermediates as well as the North. Imitation, however, only occurs in the South, and it consists of two types: efficient imitation (i.e., imitating Northern technology with certain given match level) and inefficient imitation (i.e., imitating other domestic innovators). Thus, needless to say, the Southern government, who will be in the position to choose certain kind of development strategy, faces the trade-off between imitating foreign technologies and encouraging domestic innovation.

For the North, it is shown that there is an inverted U-shaped relationship between

\footnotetext{
17 See Appendix F.

18 And just in this sense, we call the corresponding policy incentive-compatibility.
} 
its welfare and the inverse measure of Southern IPR protection. Moreover, after taking the endogenous innovation growth rate into account, we have proved that there is a U-shaped relationship between Northern equilibrium growth rate and Southern IPR protection.

On the other hand, for the South, we prove that, given the specification of lump-sum taxation, an increasing of the subsidy rate to innovation will definitely improve the welfare. And we show, in different conditions, that there always exists a corresponding optimal degree of IPR protection, and also our model points out that there are complementary relationships between those optimal IPR protection policies and the innovation subsidy policy. Furthermore, conditional on the endogenous innovation growth rate, it is argued that there is a U-shaped relationship between equilibrium growth rate and the policy of IPR protection while the equilibrium growth rate is independent of the choice of subsidy policy.

Moreover, we show that under certain weak conditions, a degree of Southern IPR protection will be optimal with respect to the welfare for both the North and the South, thereby implying the existence of an optimal degree of Southern IPR protection which is globally efficient.

Finally, we summarize the economic implications of our exploration from the following two viewpoints: firstly, for those developing countries, like China, having been involved in international trade and global competition, the corresponding policy-makers naturally face the following problem: what are the best institutional arrangements for gaining access to the knowledge (or innovation) that already exists in developed countries and meanwhile encouraging domestic production and employment of new knowledge (or innovation)? Rather, the current paper has been encouraged to translate the above circumstances requiring trade-off into our model and further our discussion has partly, if not completely, solved the above problem. Furthermore, admittedly, our ultimate goal is rapid and sustainable economic growth with widely shared and improved economic welfare. On the one hand, as is commonly known, endogenous growth theory distinguishes itself from neoclassical growth theory by incorporating knowledge (Romer, 1986, 1990; Grossman and 
Helpman, 1990), human capital (Lucas, 1988, 1993; Eicher, 1996), endogenous technological change (Romer, 1986, 1990; Grossman and Helpman, 1990; Eicher, 1996), endogenous innovation (Grossman and Helpman, 1994) and R\&D (Grossman and Helpman, 1991; Segerstrom, 1991; Aghion and Howitt, 1992; Jones, 1995; Stokey, 1995; Howitt, 1999, 2000; Segerstrom and Zolnierek, 1999) into its theoretical models, thereby leading to ongoing economic growth. Nonetheless, on the other hand, behind the theory of endogenous economic growth, there exist incentive structure problems: what encourage people to learn existing knowledge and even produce new knowledge? Why firms devote substantial resources to R\&D activities? Here, we insist, in line with North $(1971,1994)$ and Acemoglu et al (2004), that efficient institutional arrangements would form efficient incentive structure of a society, and the political and economic institutions, in consequence, are the underlying determinants of economic performance. And as is shown by our paper, optimal IPR protection is just one crucial institutional variable of many optional institutional instruments in the background of global trade.

\section{APPENDIX}

\section{A. Proof of Proposition 7.}

From (39), (41) and (45), we see that,

$$
\frac{\partial \mu_{2}^{*}}{\partial \eta_{5}^{S}}=\underbrace{\frac{\eta_{2}^{S} g^{N}-\sqrt{\left(\eta_{2}^{S} g^{N}\right)^{2}-4 \eta_{2}^{S} g^{N} \eta_{5}^{S} \rho^{S}}}{2\left(\eta_{5}^{S}\right)^{2}}}_{<0}+\underbrace{\frac{-\eta_{2}^{S} g^{N} \rho^{S}}{\eta_{5}^{S} \sqrt{\left(\eta_{2}^{S} g^{N}\right)^{2}-4 \eta_{2}^{S} g^{N} \eta_{5}^{S} \rho^{S}}}}_{>0},
$$

And so,

$$
\frac{\partial \mu_{2}^{*}}{\partial \eta_{5}^{S}}<0 \Leftrightarrow \sqrt{\left(\eta_{2}^{S} g^{N}\right)^{2}-4 \eta_{2}^{S} g^{N} \eta_{5}^{S} \rho^{S}}>\underbrace{\eta_{2}^{S} g^{N}-\eta_{5}^{S} \rho^{S}}_{<0},
$$

which always holds true. Hence, applying (41) and $\eta_{5}^{S} \equiv \eta_{1}^{S} \zeta^{S}(0)+\eta_{3}^{S}-\eta_{4}^{S}\left(1-\tau_{A^{s}}\right)$, we obtain,

$$
\frac{\partial \mu_{2}^{*}}{\partial \tau_{A^{s}}}=\frac{\partial \mu_{2}^{*}}{\partial \eta_{5}^{S}} \frac{\partial \eta_{5}^{S}}{\partial \tau_{A^{s}}}<0
$$


by (A.1). Moreover, notice by (41), (45) and (A.1) that,

$$
\frac{\partial \mu_{2}^{*}}{\partial g^{S}}=\frac{\partial \mu_{2}^{*}}{\partial \eta_{5}^{S}} \frac{\partial \eta_{5}^{S}}{\partial g^{S}}>0
$$

\section{B. Proof of Proposition 8.}

By (38) and (46), we get,

$$
\frac{\partial \mu_{3}^{*}}{\partial \eta_{6}^{S}}=\underbrace{\frac{\eta_{1}^{S} g^{S}-\sqrt{\left(\eta_{1}^{S} g^{S}\right)^{2}-4 \eta_{1}^{S} g^{S} \eta_{6}^{S} \rho^{S}}}{2\left(\eta_{6}^{S}\right)^{2}}}_{<0}+\underbrace{\frac{-4 \eta_{1}^{S} g^{S} \rho^{S}}{4 \eta_{6}^{S} \sqrt{\left(\eta_{1}^{S} g^{S}\right)^{2}-4 \eta_{1}^{S} g^{S} \eta_{6}^{S} \rho^{S}}}}_{>0}
$$

Noting that,

$$
\frac{\partial \mu_{3}^{*}}{\partial \eta_{6}^{S}}<0 \Leftrightarrow \sqrt{\left(\eta_{1}^{S} g^{S}\right)^{2}-4 \eta_{1}^{S} g^{S} \eta_{6}^{S} \rho^{S}}>\underbrace{\eta_{1}^{S} g^{S}-2 \eta_{6}^{S} \rho^{S}}_{<0},
$$

which always holds true. Therefore, by (41), (B.1) and the definition of $\eta_{6}^{s}$, we get,

$$
\frac{\partial \mu_{3}^{*}}{\partial \tau_{A^{s}}}=\frac{\partial \mu_{3}^{*}}{\partial \eta_{6}^{S}} \frac{\partial \eta_{6}^{S}}{\partial \tau_{A^{s}}}=\eta_{4}^{S} \frac{\partial \mu_{3}^{*}}{\partial \eta_{6}^{S}}<0,
$$

Furthermore, since,

$$
\eta_{6}^{S} \equiv \eta_{2}^{S} \zeta^{N}(0)+\eta_{3}^{S}-\eta_{4}^{S}\left(1-\tau_{A^{S}}\right),
$$

Then by (39), (40) and (49), we get,

$$
\frac{\partial \eta_{6}^{S}}{\partial \Theta}=\zeta^{N}(0) \frac{\partial \eta_{2}^{S}}{\partial \Theta}+\frac{\partial \eta_{3}^{S}}{\partial \Theta}=\zeta^{N}(0) \frac{\eta_{2}^{S}}{\Theta}+\eta_{8}^{S},
$$

where,

$$
\eta_{8}^{S} \equiv \partial \eta_{3}^{S} / \partial \Theta=\left(\beta^{S}\right)^{1 /\left(1-\alpha^{S}\right)} L^{S} A^{N}(0)\left[\left(\alpha^{S}\right)^{\alpha^{S} /\left(1-\alpha^{S}\right)}-\left(\alpha^{S}\right)^{1 /\left(1-\alpha^{S}\right)}\right]>0,
$$

Hence, by (39), (B.1) and (B.2),

$$
\frac{\partial \mu_{3}^{*}}{\partial \Theta}=\frac{\partial \mu_{3}^{*}}{\partial \eta_{6}^{S}} \frac{\partial \eta_{6}^{S}}{\partial \Theta}=\frac{\partial \mu_{3}^{*}}{\partial \eta_{6}^{S}}\left[1-\zeta^{N}(0)\right] \eta_{8}^{S}<0,
$$

\section{Proof of Proposition 9.}


Noting by (38), (39) and (47) that,

$$
\frac{\partial \mu_{4}^{*}}{\partial \eta_{7}^{S}}=\underbrace{\frac{\left(\eta_{1}^{S}+\eta_{2}^{S}\right) g^{S}-\sqrt{\Delta}}{2\left(\eta_{7}^{S}\right)^{2}}}_{<0}+\underbrace{\frac{-g^{S} \rho^{S}\left(\eta_{1}^{S}+\eta_{2}^{S}\right)}{\eta_{7}^{S} \sqrt{\Delta}}}_{>0},
$$

where $\Delta=\left(\eta_{1}^{S}+\eta_{2}^{S}\right)^{2}\left(g^{S}\right)^{2}-4\left(\eta_{1}^{S}+\eta_{2}^{S}\right) g^{S} \eta_{7}^{S} \rho^{S}$. Hence,

$$
\begin{aligned}
\frac{\partial \mu_{4}^{*}}{\partial \eta_{7}^{S}}<0 & \Leftrightarrow\left(\eta_{1}^{S}+\eta_{2}^{S}\right) g^{S} \sqrt{\Delta}<\Delta+2\left(\eta_{1}^{S}+\eta_{2}^{S}\right) g^{S} \eta_{7}^{S} \rho^{S} \\
& \Leftrightarrow \sqrt{\Delta}>\underbrace{\left(\eta_{1}^{S}+\eta_{2}^{S}\right) g^{S}-2 \eta_{7}^{S} \rho^{S}}_{<0},
\end{aligned}
$$

which always holds true. Thus,

$$
\frac{\partial \mu_{4}^{*}}{\partial \tau_{A^{s}}}=\frac{\partial \mu_{4}^{*}}{\partial \eta_{7}^{s}} \frac{\partial \eta_{7}^{S}}{\partial \tau_{A^{s}}}<0,
$$

by (41), (C.1) and noting the fact that $\eta_{7}^{S} \equiv \eta_{3}^{S}-\eta_{4}^{S}\left(1-\tau_{A^{S}}\right)$.

\section{Proof of Proposition 12.}

Noting that if $\zeta^{N}(0) \neq \bar{\zeta}^{N}$ and $\zeta^{S}(0) \neq \bar{\zeta}^{S}$,

$$
\frac{\partial C^{S}(0)}{\partial \mu^{S}}=-\frac{A^{S}(0)\left(1-\tau_{A^{S}}\right)}{v^{S}}\left(g^{S}\right)^{\sigma^{S}} \frac{\partial g^{S}}{\partial \mu^{S}}>0,
$$

by the following fact,

$$
b^{S}=\frac{\widehat{b^{S}(t)}}{\dot{A}^{N}(t)}=\frac{\left(g^{S}\right)^{\sigma^{S}}}{\left(\sigma^{S}+1\right) v^{S}},
$$

in (41). And from (44) we see that,

$$
\frac{\partial U^{S}}{\partial \mu^{S}}=\frac{1}{\rho^{S} C^{S}(0)} \frac{\partial C^{S}(0)}{\partial \mu^{S}}+\frac{1}{\left(\rho^{S}\right)^{2}} \frac{\partial \gamma_{C}^{S}}{\partial \mu^{S}},
$$

Furthermore, noting that, if $\zeta^{N}(0)=\bar{\zeta}^{N}, \zeta^{S}(0)=\bar{\zeta}^{S}$, and $g^{N}$ is given in (19), then,

$$
\frac{\partial \zeta^{N}(0)}{\partial \mu^{S}}=\frac{\mu^{S}}{\left(g^{N}+\mu^{S}\right)^{2}} \frac{\partial g^{N}}{\partial \mu^{S}}-\frac{g^{N}}{\left(g^{N}+\mu^{S}\right)^{2}}<0,
$$




$$
\frac{\partial \zeta^{S}(0)}{\partial \mu^{S}}=\frac{\mu^{S}}{\left(g^{S}+\mu^{S}\right)^{2}} \frac{\partial g^{S}}{\partial \mu^{S}}-\frac{g^{S}}{\left(g^{S}+\mu^{S}\right)^{2}}<0,
$$

Moreover, if $\zeta^{N}(0)=\bar{\zeta}^{N}$ and $g^{N}$ is exogenously given, thus,

$$
\frac{\partial \zeta^{N}(0)}{\partial \mu^{S}}=-\frac{g^{N}}{\left(g^{N}+\mu^{S}\right)^{2}}<0
$$

Thus, if $\zeta^{N}(0)=\bar{\zeta}^{N}$ and $\zeta^{S}(0) \neq \bar{\zeta}^{S}$, then by (39), (D.1) and (D.3),

$$
\frac{\partial C^{S}(0)}{\partial \mu^{S}}=\eta_{2}^{S} \frac{\partial \zeta^{N}(0)}{\partial \mu^{S}}-\frac{A^{S}(0)\left(1-\tau_{A^{S}}\right)}{v^{S}}\left(g^{S}\right)^{\sigma^{S}} \frac{\partial g^{S}}{\partial \mu^{S}}>0,
$$

If $\zeta^{N}(0) \neq \bar{\zeta}^{N}$ and $\zeta^{S}(0)=\bar{\zeta}^{S}$, then by (38) and (D.2),

$$
\frac{\partial C^{S}(0)}{\partial \mu^{S}}=\eta_{1}^{S} \frac{\partial \zeta^{S}(0)}{\partial \mu^{S}}-\frac{A^{S}(0)\left(1-\tau_{A^{S}}\right)}{v^{S}}\left(g^{S}\right)^{\sigma^{S}} \frac{\partial g^{S}}{\partial \mu^{S}}>0,
$$

If $\zeta^{N}(0)=\bar{\zeta}^{N}$ and $\zeta^{S}(0)=\bar{\zeta}^{S}$, then by (38), (39), (D.1), (D.2) and (D.3),

$$
\frac{\partial C^{S}(0)}{\partial \mu^{S}}=\eta_{1}^{S} \frac{\partial \zeta^{S}(0)}{\partial \mu^{S}}+\eta_{2}^{S} \frac{\partial \zeta^{N}(0)}{\partial \mu^{S}}-\frac{A^{S}(0)\left(1-\tau_{A^{S}}\right)}{v^{S}\left(g^{S}\right)^{-\sigma^{S}}} \frac{\partial g^{S}}{\partial \mu^{S}}>0,
$$

Then, the required assertion follows via combining with Proposition 10.

\section{E. Proof of Proposition 13.}

First, a comparison of (18) and (45) shows that if,

$$
\left\{\begin{array}{l}
\rho^{S}=\rho^{N}, \\
\eta_{1}^{N}=\eta_{2}^{S}, \\
\eta_{2}^{N}=\eta_{5}^{S},
\end{array}\right.
$$

Then we get $\mu_{1}^{*}=\mu_{2}^{*}$. From (14) and (39), $\eta_{1}^{N}=\eta_{2}^{S}$ implies that,

$$
\Theta=\frac{\left(\beta^{N}\right)^{1 /\left(1-\alpha^{N}\right)} L^{N}\left\{\left[\left(\alpha^{N}\right)^{2 \alpha^{N} /\left(1-\alpha^{N}\right)}-\left(\alpha^{N}\right)^{2 /\left(1-\alpha^{N}\right)}\right]-\left[\left(\alpha^{N}\right)^{\alpha^{N} /\left(1-\alpha^{N}\right)}-\left(\alpha^{N}\right)^{1 /\left(1-\alpha^{N}\right)}\right]\right\}}{\left(\beta^{S}\right)^{1 /\left(1-\alpha^{S}\right)} L^{S}\left[\left(\alpha^{S}\right)^{1 /\left(1-\alpha^{S}\right)}-\left(\alpha^{N}\right)^{\alpha^{S} /\left(1-\alpha^{S}\right)}\right]},(\mathrm{E} .
$$

which implies that $0<\Theta<1$ given $L^{S} \gg L^{N}$. From (15), (38), (40) and (41), $\eta_{2}^{N}=\eta_{5}^{S}$ means, 


$$
\begin{aligned}
& \left\{\left(\beta^{N}\right)^{1 /\left(1-\alpha^{N}\right)} L^{N}\left[\left(\alpha^{N}\right)^{\alpha^{N} /\left(1-\alpha^{N}\right)}-\left(\alpha^{N}\right)^{1 /\left(1-\alpha^{N}\right)}\right]-b^{N} g^{N}\right. \\
& \left.-\Theta\left(\beta^{S}\right)^{1 /\left(1-\alpha^{S}\right)} L^{S}\left[\left(\alpha^{S}\right)^{\alpha^{S} /\left(1-\alpha^{S}\right)}-\left(\alpha^{S}\right)^{1 /\left(1-\alpha^{S}\right)}\right]\right\} \frac{A^{N}(0)}{A^{S}(0)} \\
& =\left(\beta^{S}\right)^{1 /\left(1-\alpha^{S}\right)} L^{S}\left\{\left[\left(\alpha^{S}\right)^{2 \alpha^{S} /\left(1-\alpha^{S}\right)}-\left(\alpha^{S}\right)^{2 /\left(1-\alpha^{S}\right)}\right]\right. \\
& \left.-\left[\left(\alpha^{S}\right)^{\alpha^{S} /\left(1-\alpha^{S}\right)}-\left(\alpha^{S}\right)^{1 /\left(1-\alpha^{S}\right)}\right]\right\} \zeta^{S}(0)+\left(\beta^{S}\right)^{1 /\left(1-\alpha^{S}\right)} L^{S} \\
& \times\left[\left(\alpha^{S}\right)^{\alpha^{S} /\left(1-\alpha^{S}\right)}-\left(\alpha^{S}\right)^{1 /\left(1-\alpha^{S}\right)}\right]-b^{S} g^{S}\left(1-\tau_{A^{S}}\right),
\end{aligned}
$$

Since $\eta_{1}^{N}=\eta_{2}^{S}$ implies that,

$$
\begin{aligned}
& \left(\beta^{N}\right)^{1 /\left(1-\alpha^{N}\right)} L^{N}\left[\left(\alpha^{N}\right)^{\alpha^{N} /\left(1-\alpha^{N}\right)}-\left(\alpha^{N}\right)^{1 /\left(1-\alpha^{N}\right)}\right] \\
& +\Theta\left(\beta^{S}\right)^{1 /\left(1-\alpha^{S}\right)} L^{S}\left[\left(\alpha^{S}\right)^{1 /\left(1-\alpha^{S}\right)}-\left(\alpha^{S}\right)^{\alpha^{S} /\left(1-\alpha^{S}\right)}\right] \\
& =\left(\beta^{N}\right)^{1 /\left(1-\alpha^{N}\right)} L^{N}\left[\left(\alpha^{N}\right)^{2 \alpha^{N} /\left(1-\alpha^{N}\right)}-\left(\alpha^{N}\right)^{2 /\left(1-\alpha^{N}\right)}\right],
\end{aligned}
$$

Substituting (E.3) into (E.2) yields,

$$
\begin{aligned}
& \left\{\left(\beta^{N}\right)^{1 /\left(1-\alpha^{N}\right)} L^{N}\left[\left(\alpha^{N}\right)^{2 \alpha^{N} /\left(1-\alpha^{N}\right)}-\left(\alpha^{N}\right)^{2 /\left(1-\alpha^{N}\right)}\right]-b^{N} g^{N}\right\} \frac{A^{N}(0)}{A^{S}(0)} \\
& =\left(\beta^{S}\right)^{1 /\left(1-\alpha^{S}\right)} L^{S}\left\{\left[\left(\alpha^{S}\right)^{2 \alpha^{S} /\left(1-\alpha^{S}\right)}-\left(\alpha^{S}\right)^{2 /\left(1-\alpha^{S}\right)}\right]\right. \\
& \left.-\left[\left(\alpha^{S}\right)^{\alpha^{S} /\left(1-\alpha^{S}\right)}-\left(\alpha^{S}\right)^{1 /\left(1-\alpha^{S}\right)}\right]\right\} \zeta^{S}(0)+\left(\beta^{S}\right)^{1 /\left(1-\alpha^{S}\right)} L^{S} \\
& \times\left[\left(\alpha^{S}\right)^{\alpha^{S} /\left(1-\alpha^{S}\right)}-\left(\alpha^{S}\right)^{1 /\left(1-\alpha^{S}\right)}\right]-b^{S} g^{S}\left(1-\tau_{A^{S}}\right),
\end{aligned}
$$

In particular, as $\zeta^{S}(0) \rightarrow 1$, (E.4) can be rewritten as,

$$
\frac{A^{N}(0)}{A^{S}(0)}=\frac{\left(\beta^{S}\right)^{1 /\left(1-\alpha^{S}\right)} L^{S}\left[\left(\alpha^{S}\right)^{2 \alpha^{S} /\left(1-\alpha^{S}\right)}-\left(\alpha^{S}\right)^{2 /\left(1-\alpha^{S}\right)}\right]-b^{S} g^{S}\left(1-\tau_{A^{S}}\right)}{\left(\beta^{N}\right)^{1 /\left(1-\alpha^{N}\right)} L^{N}\left[\left(\alpha^{N}\right)^{2 \alpha^{N} /\left(1-\alpha^{N}\right)}-\left(\alpha^{N}\right)^{2 /\left(1-\alpha^{N}\right)}\right]-b^{N} g^{N}},
$$

\section{F. Proof of Proposition 14.}

First, a comparison of (18) and (47) shows that if,

$$
\left\{\begin{array}{l}
\rho^{S}=\rho^{N}, \\
\eta_{1}^{N}=\eta_{1}^{S}+\eta_{2}^{S}, \\
\eta_{2}^{N}=\eta_{7}^{S} \equiv \eta_{3}^{S}-\eta_{4}^{S}\left(1-\tau_{A^{S}}\right),
\end{array}\right.
$$


Then we get $\mu_{1}^{*}=\mu_{4}^{*}$. From (15), (40) and (41), $\eta_{2}^{N}=\eta_{7}^{S}$ implies that,

$$
\begin{aligned}
& \left\{\left(\beta^{N}\right)^{1 /\left(1-\alpha^{N}\right)} L^{N}\left[\left(\alpha^{N}\right)^{\alpha^{N} /\left(1-\alpha^{N}\right)}-\left(\alpha^{N}\right)^{1 /\left(1-\alpha^{N}\right)}\right]-b^{N} g^{N}\right. \\
& \left.-\Theta\left(\beta^{S}\right)^{1 /\left(1-\alpha^{S}\right)} L^{S}\left[\left(\alpha^{S}\right)^{\alpha^{S} /\left(1-\alpha^{S}\right)}-\left(\alpha^{S}\right)^{1 /\left(1-\alpha^{S}\right)}\right]\right\} \frac{A^{N}(0)}{A^{S}(0)} \\
& =\left(\beta^{S}\right)^{1 /\left(1-\alpha^{S}\right)} L^{S}\left[\left(\alpha^{S}\right)^{\alpha^{S} /\left(1-\alpha^{S}\right)}-\left(\alpha^{S}\right)^{1 /\left(1-\alpha^{S}\right)}\right]-b^{S} g^{S}\left(1-\tau_{A^{S}}\right),
\end{aligned}
$$

Moreover, from (14), (38) and (39), $\eta_{1}^{N}=\eta_{1}^{S}+\eta_{2}^{S}$ implies that,

$$
\begin{aligned}
& -\Theta\left(\beta^{S}\right)^{1 /\left(1-\alpha^{S}\right)} L^{S}\left[\left(\alpha^{S}\right)^{\alpha^{S} /\left(1-\alpha^{S}\right)}-\left(\alpha^{S}\right)^{1 /\left(1-\alpha^{S}\right)}\right] \frac{A^{N}(0)}{A^{S}(0)} \\
& =\left(\beta^{N}\right)^{1 /\left(1-\alpha^{N}\right)} L^{N}\left\{\left[\left(\alpha^{N}\right)^{2 \alpha^{N} /\left(1-\alpha^{N}\right)}-\left(\alpha^{N}\right)^{2 /\left(1-\alpha^{N}\right)}\right]\right. \\
& \left.-\left[\left(\alpha^{N}\right)^{\alpha^{N} /\left(1-\alpha^{N}\right)}-\left(\alpha^{N}\right)^{1 /\left(1-\alpha^{N}\right)}\right]\right\} \frac{A^{N}(0)}{A^{S}(0)}-\left(\beta^{S}\right)^{1 /\left(1-\alpha^{S}\right)} L^{S} \\
& \times\left\{\left[\left(\alpha^{S}\right)^{2 \alpha^{S} /\left(1-\alpha^{S}\right)}-\left(\alpha^{S}\right)^{2 /\left(1-\alpha^{S}\right)}\right]-\left[\left(\alpha^{S}\right)^{\alpha^{S} /\left(1-\alpha^{S}\right)}-\left(\alpha^{S}\right)^{1 /\left(1-\alpha^{S}\right)}\right]\right\},
\end{aligned}
$$

Inserting (F.2) into (F.1) produces,

$$
\frac{A^{N}(0)}{A^{S}(0)}=\frac{\left(\beta^{S}\right)^{1 /\left(1-\alpha^{S}\right)} L^{S}\left[\left(\alpha^{S}\right)^{2 \alpha^{S} /\left(1-\alpha^{S}\right)}-\left(\alpha^{S}\right)^{2 /\left(1-\alpha^{S}\right)}\right]-b^{S} g^{S}\left(1-\tau_{A^{S}}\right)}{\left(\beta^{N}\right)^{1 /\left(1-\alpha^{N}\right)} L^{N}\left[\left(\alpha^{N}\right)^{2 \alpha^{N} /\left(1-\alpha^{N}\right)}-\left(\alpha^{N}\right)^{2 /\left(1-\alpha^{N}\right)}\right]-b^{N} g^{N}},
$$

\section{REFERENCES}

Acemoglu, D., S. Johnson and J. Robinson, 2004. Institutions as the Fundamental Cause of Long-Run Growth. NBER Working Paper No. 10481.

Aghion, P. and P. Howitt, 1992. A Model of Growth Through Creative Destruction. Econometrica 60, 323-351.

Arrow, K. J., 1962. The Economic Implications of Learning by Doing. Review of Economic Studies 29, 155-173.

Canzoneri, M. B., R. E. Cumby and B. T. Diba, 2005. The need for international policy coordination: what's old, what's new, what's yet to come? Journal of International Economics 66, 363-384.

Chen, Y. and T. Puttitanun 2005. Intellectual property rights and innovation in developing countries. Journal of Development Economics 78, 474- 493.

Chin, J. and G. M. Grossman 1990. Intellectual Property Rights and North-South Trade, in R.W. 
Jones and A.O. Krueger, eds., The Political Economy of International Trade, Cambridge MA: Basil Blackwell Publishers.

Chu, A.C., G. Cozzi and S. Galli 2011. Innovating like China: a theory of stage-dependent intellectual property rights. MPRA Paper No. 30553.

Davidson, C. and P. Segerstrom, 1998. R\&D Subsidies and Economic Growth. RAND Journal of Economics 29, 548-577.

Deardorff, A. V., 1992. Welfare Effects of Global Patent Protection. Economica 59, 35-51.

Devereux, M. B. and C. Engel, 2003. Monetary Policy in the Open Economy Revisited: Price Setting and Exchange-Rate Flexibility. Review of Economic Studies 70, 765-783.

Eicher, T. S., 1996. Interaction Between Endogenous Human Capital and Technological Change. Review of Economic Studies 63, 127-144.

Furukawa, Y. 2007. The protection of intellectual property rights and endogenous growth: Is stronger always better? Journal of Economic Dynamics and Control 31, 3644-3670.

Glass, A.J., 1997. Product Cycles and Market Penetration. International Economic Review 38, 865-891.

Grossman, G. M. and E. Helpman, 1990. Comparative Advantage and Long-Run Growth. American Economic Review 80, 796-815.

Grossman, G. M. and E. Helpman, 1991. Quality Ladders in the Theory of Growth. Review of Economic Studies 58, 43-61.

Grossman, G. M. and E. Helpman, 1994. Endogenous Innovation in the Theory of Growth. Journal of Economic Perspectives 8, 23-44.

Grossman, G. M. and E. L.-C. Lai. 2004. International Protection of Intellectual Property. American Economic Review 94, 1635-1653.

Görg, H. and E. Strobl, 2007. The Effect of R\&D Subsidies on Private R\&D. Economica 74, 215-234.

Gould, D. M. and W. C. Gruben, 1996. The role of intellectual property rights in economic growth. Journal of Development Economics 48, 323-350.

Haaland, J.I. and H. J. Kind, 2008. R\&D policies, trade and process innovation. Journal of International Economics 74, 170-187.

Helpman, E. 1993. Innovation, Imitation, and Intellectual Property Rights. Econometrica 61, 1247-1280.

Horii, R. and T. Iwaisako 2007. Economic Growth with Imperfect Protection of Intellectual Property Rights. Journal of Economics 90, 45-85.

Howitt, P., 1999. Steady Endogenous Growth with Population and R \& D Inputs Growing. Journal of Political Economy 107, 715-730.

Howitt, P., 2000. Endogenous Growth and Cross-Country Income Differences. American Economic Review 90, 829-846.

Jones, C. I., 1995. R \& D-Based Models of Economic Growth. Journal of Political Economy 103, 759-784.

Kehoe, P.J., 1989. Policy Cooperation Among Benevolent Governments May Be Undesirable. Review of Economic Studies 56, 289-296.

Kwan, Y.K. and E. L. -C. Lai 2003. Intellectual property rights protection and endogenous economic growth. Journal of Economic Dynamics and Control 27, 853-873.

Levin, R. C., 1978. Technical Change, Barriers to Entry, and Market Structure. Economica 45, 
347-361.

Lucas, R. E., Jr., 1988. On the Mechanics of Economic Development. Journal of Monetary Economics 22, 3-42.

Lucas, R. E., Jr., 1993. Making a Miracle. Econometrica 61, 251-272.

Maggi, G., 1999. The Role of Multilateral Institutions in International Trade Cooperation. American Economic Review 89, 190-214.

Muniagurria, M.E. and N. Singh, 1997. Foreign Technology, Spillovers, and R \& D Policy. International Economic Review 38, 405-430.

North, D. C., 1971. Institutional Change and Economic Growth. Journal of Economic History 31, 118-125.

North, D. C., 1994. Economic Performance Through Time. American Economic Review 84, 359-368.

Romer, P. M., 1986. Increasing Returns and Long-Run Growth. Journal of Political Economy 94, 1002-1037.

Romer, P. M., 1990. Endogenous Technological Change. Journal of Political Economy 98, S71-S102.

Segerstrom, P. S., 1991. Innovation, Imitation, and Economic Growth. Journal of Political Economy 99, 807-827.

Segerstrom, P. S., 1998. Endogenous Growth without Scale Effects. American Economic Review 88, 1290-1310.

Segerstrom, P. S. and J. M. Zolnierek, 1999. The R\&D Incentives of Industry Leaders. International Economic Review 40, 745-766.

Schneider, P.H., 2005. International trade, economic growth and intellectual property rights: A panel data study of developed and developing countries. Journal of Development Economics 78, 529-547.

Scotchmer, S., 1991. Standing on the Shoulders of Giants: Cumulative Research and the Patent Law. Journal of Economic Perspectives 5, 29-41.

Spence, M., 1984. Cost Reduction, Competition, and Industry Performance. Econometrica 52, 101-122.

Spencer, B.J. and J. A. Brander, 1983. International R\&D Rivalry and Industrial Strategy. Review of Economic Studies L, 707-722.

Stein, J.C., 1997. Waves of Creative Destruction: Firm-Specific Learning-by-Doing and the Dynamics of Innovation. Review of Economic Studies 64, 265-288.

Stokey, N. L., 1995. R\&D and Economic Growth. Review of Economic Studies 62, 469-489.

Young, A., 1993. Invention and Bounded Learning by Doing. Journal of Political Economy 101, 443-472. 